\title{
Experimental evaluation of the WiMAX downlink physical layer in high-mobility scenarios
}

\author{
Pedro Suárez-Casal , José Rodríguez-Piñeiro, José A García-Naya and Luis Castedo
}

\begin{abstract}
The experimental evaluation of the WiMAX downlink physical layer in high-mobility scenarios is extremely difficult to carry out because it requires the realization of measurement campaigns with expensive fast-moving vehicles. In this work, however, we succeeded in doing such an experimental evaluation with a low-mobility vehicle. The key idea is the enlargement of the symbol period prior to its transmission over the air. Such enlargement reduces the frequency spacing between the orthogonal frequency division multiplexing (OFDM) subcarriers in WiMAX transmissions and hence induces significant inter-carrier interference $(\mathrm{ICl})$ on the received signals. The performance impact of such $I C l$ in terms of error vector magnitude (EVM) and throughput is analyzed under different conditions like the use of multiple antennas, the placement of the receive antennas, or the accuracy of the channel information to adapt the transmission rate.
\end{abstract}

Keywords: ICl; High-speed; WiMAX; MIMO

\section{Introduction}

Existing mobile communication networks are primarily designed for low user speeds below $15 \mathrm{~km} / \mathrm{h}$. Nowadays, however, there is an increased number of wireless terminals mounted on high-speed vehicles such as cars, trains, buses, subways, or airplanes. High-mobility communication networks (HMCN) aim at interconnecting such terminals to convey information not only for human users but also for machines to send all sorts of command, control, and safety information.

WiMAX is a communication standard suitable for the provision of wireless broadband connectivity. WiMAX is a term coined by the WiMAX Forum to promote the interoperability between the IEEE 802.16 family of wireless communication standards. WiMAX is the first commercially available and deployed technology for delivering mobile fourth generation (4G) services. IEEE 802.16 standardization activities started in 1999 and were the first ones to address broadband for wireless metropolitan area networks. Although long-term evolution (LTE)

*Correspondence: pedro.scasal@udc.es

Department of Electronics and Systems, University of A Coruña, Facultade de Informática, Campus de Elviña, A Coruña 15071, Spain is currently being more widely used by $4 \mathrm{G}$ mobile network operators, specially in Europe and the United States, there is still a significant amount of network operators based on WiMAX, specially in Asia [1]. More specifically, WiMAX is being used in high-mobility scenarios like the metropolitan transportation system of Japan [1]. In addition, the WiMAX Forum has recently instituted an Aviation Working Group within its organizational structure to collaborate on the adaptation of WiMAX to the specific needs of the aviation community [2].

The physical layer (PHY) of the WiMAX radio interface uses orthogonal frequency division multiplexing (OFDM) as the modulation scheme. OFDM is particularly suitable to carry data over broadband frequency-selective channels because it allows for a low-cost channel equalization. OFDM waveforms, on the contrary, are rather sensitive when transmitting over time-selective channels such as those encountered in high-mobility scenarios. Time-selectivity causes the OFDM subcarriers to loose their orthogonality property and produces inter-carrier interference (ICI). Time-selectivity is particularly harmful when considering complex scenarios with multiple antennas and/or multiple users.

\section{是 Springer}


Different techniques have been proposed in the literature to mitigate the effects of ICI. More specifically, issues like pilot pattern design [3], window design [4], channel estimation [5], and channel equalization have been thoroughly studied.

Basis expansion models (BEMs) have been extensively used to capture the double selectivity of the wireless channel and to implement cost-effective channel estimation and equalization schemes [6]. The rationale under BEM is the efficient representation of either the impulse or the frequency response of a channel by means of a linear combination of some basis vectors. Different BEMs have been proposed to be used under doubly-selective channels, such as the complex exponential BEM (CEBEM) [7], polynomial BEM (P-BEM) [8,9], discrete prolate spheroidal BEM (DPS-BEM) [10], and Karhunen-Loève BEM (KL-BEM) [11,12]. Regarding pilot patterns for OFDM under ICI conditions, the Kronecker delta model, where guard subcarriers are allocated around pilot subcarriers, has been proposed as an optimal design to minimize channel estimation errors $[13,14]$. A detailed review of the algorithms and techniques to combat double-selectivity of the wireless channel can be found in [15].

Communication standards such as WiMAX or LTE define the pilot structure to be used by the elements in the network. Such pilot structures typically do not correspond to those assumed in many ICI estimation and equalization techniques proposed in the literature. In this work, we focus on specific algorithms which do not depend on such assumptions $[12,16,17]$. These methods typically estimate the ICI from a set of consecutive frequency response estimations and exploit time variations per subcarrier to estimate its spreading.

Experimental performance evaluations of $4 \mathrm{G}$ technologies in high-mobility situations are scarce in the literature due to the huge difficulties of carrying out experiments in such scenarios. In this work, we follow an approach where the time-selective wireless channels of high-mobility situations are recreated from experiments carried out at low speeds and hence are more cost-efficient to implement [18]. The approach consists of time-interpolating OFDM symbols prior to its transmission over-the-air (OTA), which leads to a reduction of the bandwidth of the whole OFDM signal. This produces OFDM waveforms which convey exactly the same information as the original one but with a reduced subcarrier spacing, hence artificially increasing the waveform sensitivity to ICI. At reception, and prior to its demodulation, the interpolation operation is inverted via a simple decimation operation. The resulting OFDM symbols are affected by ICI similarly as if they were transmitted over a high-mobility wireless channel. In fact, interpolating the original signal by a factor $I$ will affect the transmitted signal similar to what would happen if it were transmitted at $I$ times the original speed.
In this paper, we extend preliminary results obtained using this technique and already reported in $[18,19]$. The main contributions of this paper are as follows:

- Evaluation of ICI estimation and cancellation algorithms with experimental measurements of multiple-input multiple-output (MIMO) transmissions under time-selective conditions. These results are compared to simulations with comparable model parameters.

- Experimental study of the impact of ICI and feedback delay on performance metrics such as throughput.

- Improved measurement methodology with respect to that used in previous works $([18,19])$. Instead of carrying out a dedicated measurement for each emulated speed value, the signals corresponding to all emulated speeds (time interpolation factors) are transmitted sequentially right after each other, thus making possible their latter comparison under similar conditions. Additionally, two receivers are used simultaneously, and therefore outdoor-to-outdoor as well as outdoor-to-indoor measurements are recorded under similar conditions.

This work focuses on OFDM waveforms based on the WiMAX physical layer. Howerver, note that the experimental methodology also applies to other OFDM-based transmissions, such as those used in LTE. Although the results are obtained for a particular standard, this work provide hints on the behavior of other OFDM-based transmissions on high-mobility conditions and on the performance of the ICI cancellation techniques in different scenarios.

\section{Signal model}

We consider the transmission of MIMO-OFDM symbols synthesized according to the PHY layer specifications of the IEEE 802.16e (Mobile WiMAX) standard. We assume OFDM symbols with $N$ subcarriers and a cyclic prefix of $N_{g}$ samples are transmitted. In total, each OFDM symbol occupies $N_{t}=N+N_{g}$ samples. OFDM symbols are grouped in frames of $K$ consecutive symbols, including a symbol reserved as a preamble. We also assume multiple antennas at transmission and reception, i.e., MIMOOFDM transmissions. The number of transmit and receive antennas is $M_{T}$ and $M_{R}$, respectively. OFDM symbols are spatially multiplexed over the $M_{T}$ transmit antennas except the preamble which is transmitted only through the first antenna.

Let $\mathbf{s}_{k}^{(m)} \in \mathbb{C}^{N \times 1}, k=1, \ldots, K, m=1, \ldots, M_{T}$ be the column vector that represents the $N$ complex-valued information symbols transmitted in the kth OFDM symbol over the $m$ th antenna. Such vectors contain data, pilot, and guard symbols. Similarly, $\mathbf{x}_{k}^{(m)} \in \mathbb{C}^{N_{t} \times 1}$ contains 
the $N_{t}$ samples corresponding to the $k$ th OFDM symbol transmitted over the $m$ th antenna.

Elaborating the signal model of a MIMO-OFDM system, the discrete-time representation of the transmitted MIMO-OFDM symbols is:

$$
\mathbf{x}_{k}=\left(\mathbf{I}_{M_{T}} \otimes \mathbf{G}_{1} \mathbf{F}^{H}\right) \mathbf{s}_{k}, \quad k=1, \ldots, K,
$$

where $\mathbf{s}_{k}=\left[\mathbf{s}_{k}^{(1)^{T}}, \mathbf{s}_{k}^{(2)}, \cdots, \mathbf{s}_{k}^{\left(M_{T}\right)^{T}}\right]^{T}$ is the $N M_{T} \times$ 1 column vector containing the information, pilot, and guard symbols transmitted in the $k$ th MIMO-OFDM symbol; $\mathbf{F}$ is the standard $N \times N$ discrete Fourier transform (DFT) matrix; $\mathbf{G}_{1}$ is a $N_{t} \times N$ matrix which appends the $N_{g}$ samples of the cyclic prefix; $\otimes$ denotes the Kronecker product; $\mathbf{I}_{M_{T}}$ is the $M_{T} \times M_{T}$ identity matrix; and $\mathbf{x}_{k}=$ $\left[\mathbf{x}_{k}^{(1)}{ }^{T}, \mathbf{x}_{k}^{(2)}{ }^{T}, \cdots, \mathbf{x}_{k}^{\left(M_{T}\right)^{T}}\right]^{T}$ is the $N_{t} M_{T} \times 1$ column vector with the samples of the $k$ th MIMO-OFDM symbol. We assume the samples in $\mathbf{x}_{k}^{(m)}$ are serially transmitted over the $m$ th antenna at a sampling rate $F_{s}=1 / T_{s}$ where $T_{s}$ is the sampling period.

The information symbol vectors are constructed as $\mathbf{s}_{k}^{(m)}=\mathbf{P}_{k}^{(m)} \mathbf{p}_{k}^{(m)}+\mathbf{D}_{k}^{(m)} \mathbf{d}_{k}^{(m)}$ where $\mathbf{p}_{k}^{(m)}$ is a $P \times 1$ vector containing the pilot symbols in the $k$ th OFDM symbol transmitted over the $m$ th transmit antenna, whereas $\mathbf{P}_{k}^{(m)}$ is the $N \times P$ matrix that defines the positions of the pilots in such a symbol. Similarly, $\mathbf{d}_{k}^{(m)}$ is the $D \times 1$ vector containing the data symbols, and $\mathbf{D}_{k}^{(m)}$ is the $N \times D$ matrix that defines their positions. Data symbols are the output of a quadrature amplitude modulation (QAM) constellation mapper whose inputs are the channel encoded source bits. Note that $P+D<N$, with $N-(P+D)$ being the number of guard subcarriers. Matrices $\mathbf{P}_{k}^{(m)}$ and $\mathbf{D}_{k}^{(m)}$ consist of ones and zeros only and are designed so that data and pilots are assigned to different subcarriers. According to the Mobile WiMAX standard, pilot subcarriers allocated in the $m$ th antenna are set to 0 in all other antennas.

We next assume that the previous OFDM waveforms are transmitted over a time-varying MIMO channel. Such a channel is represented by:

$$
h^{i, j}(t, \tau)=\sum_{l=1}^{L} h_{l}^{i, j}(t) \delta\left(\tau-\tau_{l}^{i, j}\right), i=1, \ldots, M_{R} \text { and } j=1, \ldots, M_{T},
$$

where $h^{i, j}(t, \tau)$ is the channel impulse response between the $j$ th transmit antenna and $i$ th receive antenna, while $h_{l}^{i, j}(t)$ and $\tau_{l}^{i, j}$ are the $l$ th path gain and delay of $h^{i, j}(t, \tau)$, respectively. Elaborating on the discrete-time signal model of a MIMO-OFDM system, the MIMO channel during the transmission of the $k$ th OFDM symbol is represented by the block matrix:

$$
\mathbf{H}_{k}=\left[\begin{array}{cccc}
\mathbf{H}_{k}^{1,1} & \mathbf{H}_{k}^{1,2} & \ldots & \mathbf{H}_{k}^{1, M_{T}} \\
\mathbf{H}_{k}^{2,1} & \mathbf{H}_{k}^{2,2} & \cdots & \mathbf{H}_{k}^{2, M_{T}} \\
\cdots & \cdots & \cdots & \cdots \\
\mathbf{H}_{k}^{M_{R}, 1} & \mathbf{H}_{k}^{M_{R}, 2} & \cdots & \mathbf{H}_{k}^{M_{R}, M_{T}}
\end{array}\right],
$$

where $\mathbf{H}_{k}^{i, j} \in \mathbb{C}^{N_{t} \times N_{t}}$ are matrices representing the channel impulse response between the $(i, j)$ antenna pair whose entries are:

$\mathbf{H}_{k}^{i, j}(r, s)=h^{i, j}\left(\left((k-1) N_{t}+N_{g}+r-1\right) T_{s}, \bmod (r-s, N) T_{s}\right)$.

Note that $\mathbf{H}_{k}^{i, m} \mathbf{x}_{k}^{(m)}$ represents the time-convolution between the signal transmitted over the $m$ th antenna and $h^{i, m}(t, \tau)$.

Having in mind the previous channel representation, the discrete-time received signal is given by:

$$
\mathbf{r}_{k}=\left(\mathbf{I}_{M_{R}} \otimes \mathbf{F G}_{2}\right) \mathbf{H}_{k} \mathbf{x}_{k}+\mathbf{w}_{k}=\mathbf{G}_{k} \mathbf{s}_{k}+\mathbf{w}_{k},
$$

where $\mathbf{G}_{2}$ is a $N \times N_{t}$ matrix which represents the removal of the cyclic prefix, $\mathbf{G}_{k}$ is a block matrix containing the channel frequency response between each antenna pair, and $\mathbf{w}_{k} \in \mathbf{C}^{N_{t} \times 1}$ is a vector of independent complexvalued Gaussian-distributed random variables with variance $\sigma_{w}^{2}$. When the channel is time invariant, all submatrices in $\mathbf{G}_{k}$ are diagonal. However, when the channel is time-variant, entries different than zero appear outside their main diagonals, hence introducing ICI between the transmitted subcarriers. In such case, Equation 5 is rewritten as:

$$
\mathbf{r}_{k}=\overline{\mathbf{G}}_{k} \mathbf{s}_{k}+\mathbf{z}_{k}+\mathbf{w}_{k}
$$

where $\overline{\mathbf{G}}_{k}$ is a block matrix with the main diagonals of the submatrices of $\mathbf{G}_{k}$ and $\mathbf{z}_{k}=\left(\mathbf{G}_{k}-\overline{\mathbf{G}}_{k}\right) \mathbf{s}_{k}$ represents the ICI in the received signal. Note that in multi-antenna systems, ICI occurs not only among subcarriers but also among different transmit antennas.

When transmitting MIMO-OFDM symbols over timevarying channels, the amount of ICI relates to the normalized Doppler spread given by $D_{n}=f_{d} T$, where $f_{d}$ is the maximum Doppler frequency and $T=N T_{s}$ is the duration of an OFDM symbol excluding the cyclic prefix. According to our previous proposal [18], it is possible to adjust the parameter $T$ by time interpolation by a factor $I$, yielding an OFDM symbol duration $T^{I}=I T_{s} N$. Therefore, the normalized Doppler spread impacting the time-interpolated OFDM signal is:

$$
D_{n}^{I}=f_{d} T^{I}=f_{d} I T_{s} N=\frac{T_{s} N I f_{c} v}{c}=\frac{T_{s} N f_{c}}{c} v^{I},
$$

with $f_{c}$ the carrier frequency, $c$ the speed of light, and $v^{I}=$ $I v$ the emulated speed as a result of an actual measurement speed $v$ and a time-interpolation factor $I$. Consequently, enlarging the symbol length $T^{I}$ allows for the emulation of 
a velocity $v^{I}$, which is $I$ times higher than the actual speed of the receiver, namely $v$.

Figure 1 shows the graphical representation of a measurement setup designed according to the previous premises. Interpolating the MIMO-OFDM waveforms prior to its transmission allows for emulating highvelocity conditions while conducting low-velocity experiments. Due to the interpolation step, signals over the air will suffer from severe ICI degradation, although the maximum Doppler frequency is low.

It should be noticed that interpolation does not allow for a perfect recreation of high-mobility channels because the signals over-the-air have a reduced bandwidth and are less sensitive to the channel frequency selectivity.

Nevertheless, note that in this work, we are mostly interested in conducting experiments to test the performance of ICI cancellation methods in WiMAX receivers over real-world channels rather than channel equalization methods which can be tested in static experiments. Time interpolation does not reproduce the exact conditions of high mobility scenarios but provide a cost-efficient approximation to them.

\section{Receiver structure}

Figure 2 shows the block diagram of the receiver structure utilized along this work. The samples captured with the hardware equipment are first decimated to undo the interpolation applied at the transmitter to induce the ICI. The decimated samples are input to an OFDM receiver that performs frame detection, and time and fractional carrier frequency offset (CFO) estimation before the DFT; and channel estimation, ICI cancellation, data subcarriers equalization, and channel decoding after the DFT. Figure 2 shows the general structure of the receiver. The same OFDM receiver is used to obtain the experimental results and the simulation results.

The ensuing subsections present a more detailed description of each processing block represented in Figure 2.

\section{Frame detection and synchronization}

Frame detection is carried out using the correlation properties of the preamble symbol. WiMAX defines a preamble symbol with pilot subcarriers generated from a pseudo-noise sequence modulated as binary phase-shift keying (BPSK), with a spacing of two guard subcarriers between them. This structure leads to a threefold repetition in the time domain, which can be exploited for frame detection and time and fractional carrier frequency offset (CFO) estimation. Integer frequency shifts are corrected after the DFT by performing a cross-correlation between the differential sequences of the transmitted and received preamble pilot sequences $[20,21]$.

\section{$\mathrm{ICl}$ estimation}

The frequency response matrix $\mathbf{G}_{k}$ in the received MIMOOFDM signal model given by Equation 5 needs to be estimated. We start determining a noisy frequency response estimation as a least squares (LS) estimation on the pilot subcarriers, affected by the interferences $\mathbf{z}_{k}$ and $\mathbf{w}_{k}$, followed by a linear minimum mean squared error (LMMSE) interpolation of the channel coefficients on the data subcarriers. Recall that pilot subcarriers are transmitted over disjoint sets of subcarriers at different antennas.

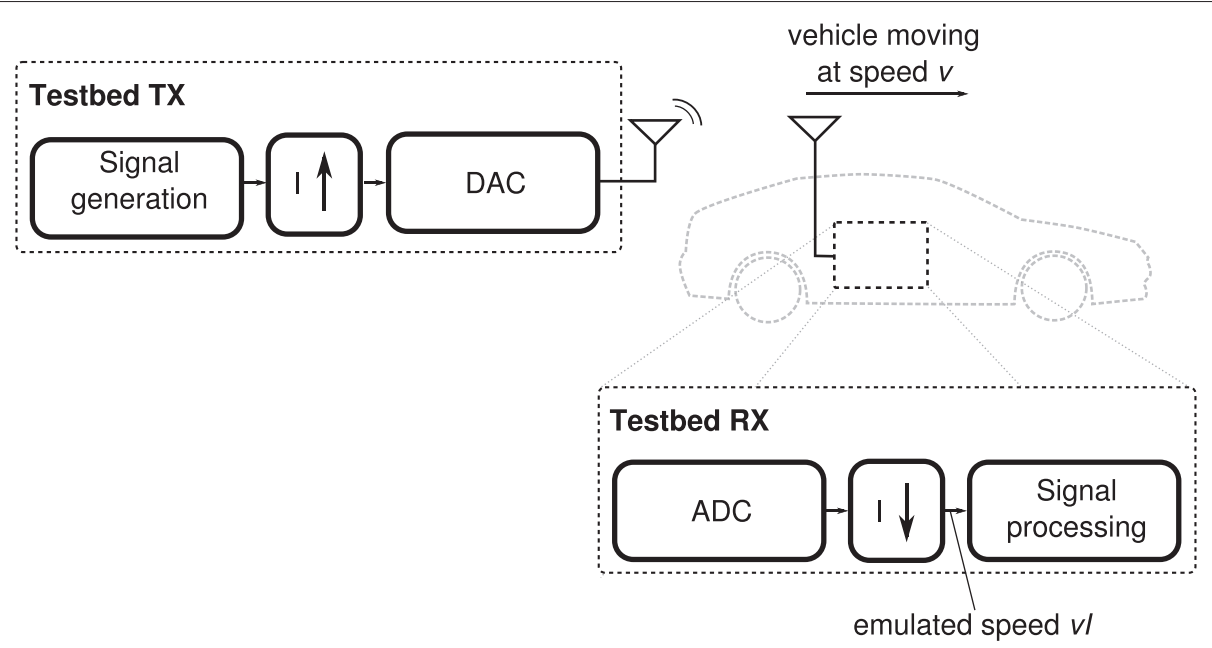

Figure 1 Block diagram of the outdoor-to-outdoor measurement setup. The transmit antennas are placed outdoors, and the receive antennas are installed outside the car on its roof. The interpolator at the transmitter side and the decimator at the receiver side enable the recreation of high-speed conditions during low-speed experiments. The corresponding outdoor-to-indoor setup is basically the same. The only difference is that receiving antennas are placed inside the car instead of outside. 


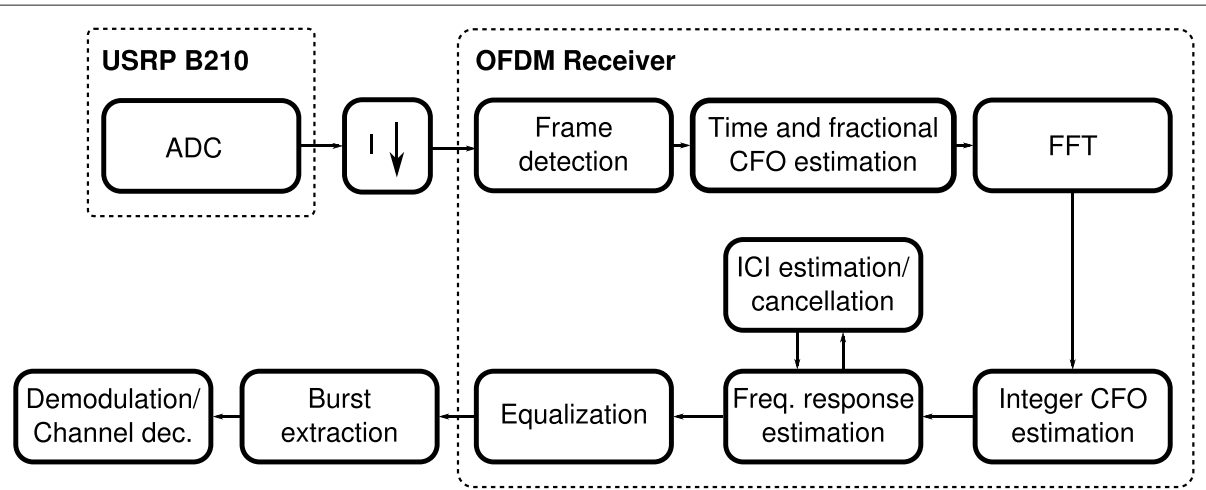

Figure 2 Block diagram of the receiver structure with decimation and the OFDM receiver with Inter-Carrier Interference (ICI) cancellation. The OFDM receiver is the same for the experimental and the simulation results.

Assuming spatially uncorrelated channels, this estimation can be done independently for each transmit-receive antenna pair. For simplicity reasons, antenna indices will be dropped in the following expressions.

The previous frequency response estimations can be expressed as:

$$
\hat{\mathbf{g}}_{k}=\operatorname{Adiag}\left\{\mathbf{p}_{k}\right\}^{-1} \mathbf{P}_{k}^{T} \mathbf{r}_{k}
$$

where $\mathbf{A}=\mathbf{C}_{h h_{p}}\left(\mathbf{C}_{h_{p} h_{p}}+\sigma_{I}^{2} \mathbf{I}_{P}\right)^{-1}$ is a $N \times P$ matrix with the LMMSE interpolation coefficients, $\mathbf{C}_{h h_{p}}=\mathbf{P}_{k}^{T} \mathrm{E}\left\{\mathbf{g}_{k} \mathbf{g}_{k}^{T}\right\}$ and $\mathbf{C}_{h_{p} h_{p}}=\mathbf{P}_{k}^{T} E\left\{\mathbf{g}_{k} \mathbf{g}_{k}^{T}\right\} \mathbf{P}_{k}$ are submatrices of the covariance matrix of the channel frequency response, and $\hat{\mathbf{g}}_{k}$ is a $N \times 1$ vector with the estimated channel coefficients per subcarrier. The second-order statistics of the channel are estimated by using all the received pilot subcarriers in a frame. Also, the power $\sigma_{z}^{2}$ of the ICI arising from $\mathbf{z}_{k}$ in Equation 6 is estimated according to [22], and it is considered as part of $\sigma_{I}^{2}=\sigma_{w}^{2}+\sigma_{z}^{2}$. As explained below, these frequency response estimations play a fundamental role in the estimation of the ICI.

Indeed, the ICI frequency response matrix $\mathbf{G}_{k}$ can be estimated by expressing the subcarrier spreading in terms of a BEM represented by a $R N_{t} \times Q$ matrix $\mathbf{B}=$ $\left[\mathbf{B}_{1}^{T}, \mathbf{B}_{2}^{T}, \cdots, \mathbf{B}_{R}^{T}\right]^{T}$, where the dimensions of $\mathbf{B}_{r}$ are $N_{t} \times$ $Q$, with $r=1, \ldots, R$. Matrix $\mathbf{B}$ represents the basis vector for the time evolution of the channel during $R$ consecutive OFDM symbols, with $Q$ the order of the BEM. The BEM allows for expressing the frequency response matrix $\mathbf{G}_{k}$ in terms of a matrix $\mathbf{C}$ of size $Q \times N$. Using BEM, the estimate of the $r$ th symbol in the group can be written as:

$$
\hat{\mathbf{G}}_{r}=\sum_{q=1}^{Q} \mathbf{F G}_{2} \operatorname{diag}\left\{\mathbf{b}_{r, q}\right\} \mathbf{G}_{1} \mathbf{F}^{H} \operatorname{diag}\left\{\hat{\mathbf{c}}_{q}\right\},
$$

where $\mathbf{b}_{r, q}$ is the $q$ th column of $\mathbf{B}_{r}, \mathbf{G}_{1}$ and $\mathbf{G}_{2}$ are the matrices which append and remove the cyclic prefix in Equation 5 , and $\hat{\mathbf{c}}_{q}$ is a $N \times 1$ vector with the columns of the estimate $\hat{\mathbf{C}}^{T}$.
We next define the $N \times R$ matrix $\hat{\mathbf{J}}_{r}=$ $\left[\hat{\mathbf{g}}_{r}, \hat{\mathbf{g}}_{r+1}, \cdots, \hat{\mathbf{g}}_{r+R-1}\right]$, with $r \in[1, K-R+1]$. Such a matrix enables us to estimate the matrix $\mathbf{C}$ of BEM coefficients by means of the following LS regression [17]:

$$
\hat{\mathbf{C}}_{r}=\left(\mathbf{K}^{H} \mathbf{K}\right)^{-1} \mathbf{K}^{H} \hat{\mathbf{J}}_{r}^{T},
$$

where $\mathbf{K}$ is a $R \times Q$ matrix whose columns are obtained from $\mathbf{B}$ as:

$\mathbf{k}_{q}=\left[b_{q}\left(N_{g}+N / 2\right), b_{q}\left(N_{t}+N / 2\right), \cdots, b_{q}\left((N / 2)+(R-1) N_{t}\right)\right]$.

Finally, the estimates $\hat{\mathbf{c}}_{q}$ in Equation 9 are obtained from the columns of $\hat{\mathbf{C}}_{r}^{T}$, which provide the coefficients for the channel matrices $\mathbf{G}_{r}$ of the group.

The results in this work are all obtained by using the discrete prolate spheroidal BEM (DPS-BEM) [10]. Such a BEM is built on the Slepian sequences arising from time sequences whose energy is localized on a given frequency interval. For the purpose of ICI estimation, the frequency interval is the one corresponding to the Doppler spectrum, whose domain is bounded by the maximum Doppler frequency. Recall that the use of DPS-BEMs is equivalent to assuming a time-selective channel with a flat Doppler spectrum. Consequently, to determine the specific DPS-BEM appropriate for a given scenario, it is necessary to know the mobile speed, and more specifically, the emulated speed after interpolation [10].

\section{$\mathrm{ICl}$ cancellation and equalization}

Once the full frequency response matrices for all antenna pairs are obtained, both ICI cancellation and equalization of the received signal are done to obtain estimates of the information symbols $\mathbf{s}_{k}$. In the literature, both block interference cancellation (BIC) and sequential interference cancellation (SIC) schemes have been proposed $[15,23]$. In this work, a LMMSE SIC receiver of seven taps is used to remove the ICI and equalize the channel. Such a 
method has been chosen due to its good trade-off between computational cost and performance.

The ICI estimation algorithm described in the previous subsection works on groups of OFDM symbols. Nevertheless, it should be noticed that the estimation error inside the group is not uniform along the OFDM symbols. Lower errors are achieved in the central symbols, and this is taken into account by the equalizer implemented in our receiver. ICI cancellation and channel equalization is carried out according to the following steps:

1. Obtain the matrices $\hat{\mathbf{C}}_{r}$ for the $K-R+1$ groups in a frame, as explained in the previous subsection.

2. Remove ICI from each OFDM symbol as follows:

(a) For symbols $k \in[1, R / 2]$, ICI is suppressed with the coefficients obtained from $\hat{\mathbf{C}}_{1}$.

(b) For symbols $k \in[R / 2+1, K-R / 2]$, ICI is suppressed with the coefficients obtained from $\hat{\mathbf{C}}_{k-R / 2+1}$.

(c) For symbols $k \in[K-R / 2+1, K]$, ICI is suppressed with the coefficients obtained from $\hat{\mathbf{C}}_{K-R+1}$.

3. Obtain a new frequency response estimate from the ICI-reduced received signal and return to step 1.

As can be seen, except for the first and last symbols of the frame, ICI is estimated and cancelled for the central OFDM symbol of each set. The final equalization of the ICI-reduced signal is done by zero forcing. Detected information symbols are demapped and sent to a Viterbi decoder to obtain the information bits.

\section{Experimental setup}

The testbed employed for the experimental evaluation in this work is an upgrade of that employed in the measurement campaigns described in $[18,19]$ which, at the same time, has evolved from the one described in [24]. The testbed consists of three USRP B210 boards [25] (see Figures 3 and 4) built from the AD9361 chip [26] by Analog Devices, which supports a continuous frequency coverage from $70 \mathrm{MHz}$ to $6 \mathrm{GHz}$; full-duplex MIMO operation with up to $56 \mathrm{MHz}$ of bandwidth; USB 3.0 connectivity; on-chip 12 bit analog-to-digital converters (ADC) and digital-to-analog converters (DAC) up to $61.44 \mathrm{M}$ sample/s; automatic gain control; and configurable transmit and receive gain values.

A single board is used in continuous transmit-only mode for implementing the base station transmitter for the downlink. The base station is equipped with two Mini-Circuits TVA-11-422 high-power amplifiers [27], two Interline SECTOR IS-G14-F2425-A120-V vertically polarized transmit antennas [28], and an Ubiquity AM2G15-120 cross polarized transmit antenna [29]. Notice

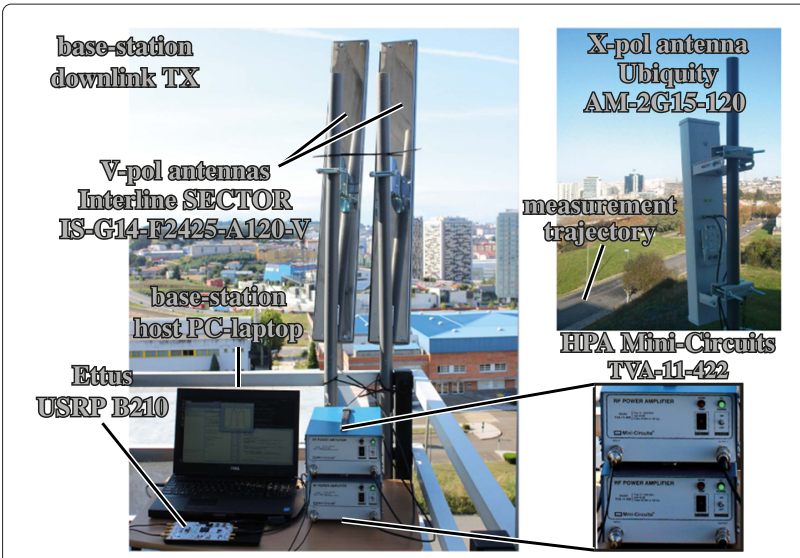

Figure 3 Base station downlink transmitter is placed outdoors. Located on the second floor of the CITIC building located in the Campus de Elviña at the University of A Coruña. Note that only one of the two vertically polarized antennas is used for $1 \times 1$ transmissions, while both cross polarized antennas are used for the $2 \times 2 \mathrm{MIMO}$ transmissions.

that a single vertically polarized antenna was used for the single-input single-output (SISO) transmissions, while the cross polarized antenna was employed for measuring the $2 \times 2$ MIMO ones.

The remaining two USRP $\mathrm{B} 210$ boards are used for implementing two different mobile receivers, both mounted on a car. The first one is connected to a couple of eRize ERZA24O-09MBR omnidirectional $9 \mathrm{dBi}$-gain antennas placed outdoors, on the roof the car. The second mobile receiver is connected to another two omnidirectional antennas placed inside the car, between the two front seats. Using both mobile receivers allows us to capture, at the same time, the signals transmitted by the base station to both outdoor and indoor receivers.

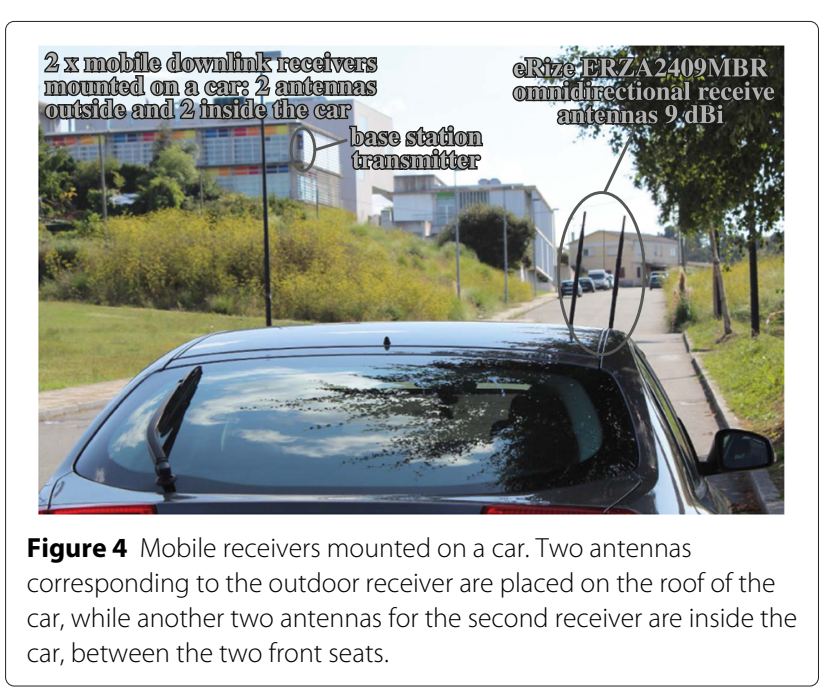


With respect to the software, we use a multi-thread receiver implemented in $\mathrm{C}++$ with Boost and using the Ettus USRP Hardware Driver (UHD) software. The main thread of the receiver is responsible for retrieving the samples coming from the USRP through the USB 3.0 bus and store them in a set of buffers in the main memory of the host laptop. The second thread reads the samples from such buffers and saves them persistently in a dedicated solid-state drive. Finally, there is a low-priority thread for logging important information for documenting the measurement campaign. On the other hand, the transmitter is a single-thread process since the same signals are cyclically transmitted over-the-air. Therefore, the signals to be radiated are first stored in a temporary buffer and next transmitted in a loop to the USRP through the USB 3.0. The rest of the software was implemented in MATLAB.

For the measurements, we use the WiMAX profile corresponding to $7 \mathrm{MHz}$ of bandwidth at a sampling rate of $F_{b}=8 \mathrm{M}$ sample/s, $N=1,024$ subcarriers, and a cyclic prefix of $1 / 8\left(N_{g}=128\right)$. Table 1 summarizes the parameter values of this WiMAX profile. At the base station, once the OFDM signals were generated, they are time interpolated with the interpolation factors $I \in\{4,12,20\}$, hence producing signals with bandwidth ranging from $1.75 \mathrm{MHz}$ for $I=4$ to $350 \mathrm{kHz}$ for $I=20$. Next, the signals corresponding to each interpolation factor $I$ are scaled by a factor $\sqrt{I}$ in order that signals corresponding to all interpolation factors be transmitted with the same energy. The three time-interpolated frames are then frequencyshifted $1.2 \mathrm{MHz}$ to avoid the DC leakage at both the transmitter and the receiver. Hence, the DC subcarrier is frequency shifted $1.2 \mathrm{MHz}$ to avoid the distortion caused by the DC leakage to the subcarriers around the DC, since the subcarrier spacing is reduced and we are dealing with direct-conversion transceivers. In order to facilitate the signal-to-noise ratio (SNR) estimation at the receiver, after transmitting the three time-interpolated frames, the transmitter is kept silent during a small time lapse which allows for estimating the noise variance at the receiver.

Table 1 Parameter values of the WiMAX profile used in the experiments

\begin{tabular}{lc}
\hline Parameter & Value \\
\hline Sampling frequency & $8 \mathrm{MHz}$ \\
Useful bandwidth & $7 \mathrm{MHz}$ \\
Number of subcarriers & 1,024 \\
Number of data subcarriers & 720 \\
Number of pilot subcarriers & 120 \\
Subcarrier spacing & $7.81 \mathrm{kHz}$ \\
Cyclic prefix length & $128 \mathrm{samples}$ \\
Permutation zone & PUSC \\
\hline
\end{tabular}

Finally, all generated signals are stored in a file in the disk of the base station transmitter.

Once the transmit signals have been generated, the base station is notified and the over-the-air transmission process starts. First, the signals are read from the corresponding source file and transferred to the USRP, where they are again interpolated in the FPGA before reaching the $(\mathrm{DAC})$. Note that this interpolation stage is needed for adapting the signal sampling rate to the sampling frequency of the DAC, thus not affecting the signal bandwidth. Next, the signals are up-converted to the central frequency $f_{c}=2.6 \mathrm{GHz}$, pre-amplified inside the USRP (configured with a gain value of $60 \mathrm{~dB}$ out of $89.5 \mathrm{~dB}$ ), amplified by the two Mini-Circuits TVA-11422 high-power amplifiers (one per transmit antenna) at their maximum gain of $40 \mathrm{~dB}$, and finally radiated by the antennas (we use the vertically polarized transmit antennas for SISO transmissions, while the cross polarized ones are employed for $2 \times 2$ MIMO ones). The mean transmit power value measured at each antenna input is $+17.5 \mathrm{dBm}$ when a single transmit antenna is used. In case both transmit antennas are employed, the transmit gain is reduced to $3 \mathrm{~dB}$ per antenna to keep the total transmit power equal despite of the number of transmit antennas.

Figure 3 shows the base station placed outdoors, on the second floor of the CITIC building located in the Campus de Elviña at the University of A Coruña. It also shows the power amplifiers, the antennas (vertically or cross polarized), the USRP B210, and the laptop running the software for the base station. Looking at the picture of the crosspolarized antennas in Figure 3, one can also see part of the road traversed by the car during the measurements.

The two receivers are also built around the USRP B210 and the UHD with the software installed on two laptops, one per receiver. Notice that during the measurements, the acquired signals are persistently stored in dedicated solid state drives attached to laptop receivers, but they are not processed using the WiMAX receiver at that moment. As shown in Figure 4, the two outdoor receive antennas are sticked on the roof of the car used for measuring and they are connected directly to the USRP B210, which is powered by its corresponding laptop. The receive gain of the USRP is set to $35 \mathrm{~dB}$ (out of $73 \mathrm{~dB}$ ), ensuring a linear operation. The second receiver is completely installed inside the car used for the measurements, with the antennas between the two front seats of the car. We use another laptop for running the receive software and for persistently storing the signals acquired by the indoor receiver. Unlike the outdoor receiver, the receive gain of the indoor receiver is set to $45 \mathrm{~dB}$ to better accommodate the amplitude of the acquired signals to the range of the ADC, while ensuring that the receive signal is not distorted by the amplifiers. 
Figure 5 shows the measurement scenario, including the position of the base station and the path followed by the mobile receivers in the car. From the starting to the end points, there is a distance of $210 \mathrm{~m}$, which is traversed by the car in $25.2 \mathrm{~s}$ at a constant speed of $30 \mathrm{~km} / \mathrm{h}$.

\section{Physical layer configuration}

OFDM symbol structure of the transmitted signal follows the downlink of mobile WiMAX standard regarding subcarrier allocation, symbol mapping, and channel encoding. The frames consists of $K=25$ symbols, with the first one reserved for the preamble signal transmitted only by the first antenna. The other symbols carry the information of six bursts with the modulation and channel coding profiles: 4-QAM 1/2, 4-QAM 3/4, 16-QAM 1/2, 16-QAM 3/4, 64-QAM 1/2, and 64-QAM 3/4. Each burst spans during four consecutive OFDM symbols along all the available data subcarriers. The permutation matrices $\mathbf{P}_{k}^{(m)}$ and $\mathbf{D}_{k}^{(m)}$ are generated according to the partial usage of subcarriers (PUSC) zone with the corresponding modifications for MIMO as specified in the standard and pilot subcarriers on $\mathbf{p}_{k}^{(m)}$ generated as a boosted BPSK sequence obtained by mapping the output of a linear feedback shift register. Channel coding is performed by the convolutional encoder defined in the standard, which features tail-biting to terminate coding blocks.

Regarding the receiver, a DPS-BEM with $Q=5$ is used for ICI estimation, given its good properties to estimate ICI assuming that only the maximum Doppler frequency is known.

In order to ensure similar channel characteristics for all the interpolation factors in each measurement, a superframe consisting of four different parts is built. The first part is a small time period during which the base station is kept silent to allow for noise variance estimation at the receiver as it was already explained. The remaining three parts are built from the same mobile WiMAX frame consisting of the six burst plus the preamble that was described above. Consequently, the second part of the super-frame corresponds to the aforementioned mobile WiMAX frame interpolated in time by the factor $I=4$. Next, the third part is the same as the second one but using $I=12$, while the fourth part employs $I=20$. Finally, the super-frame is cyclically transmitted from the base station to both mobile receivers, one placed outside the car and the other installed inside.

\section{Results}

This section presents the results obtained from the measurements described above. We also carried out simulations to support the experimental results. Simulations were designed to create scenarios comparable to those obtained from the measurements. Parameters estimated from the real-data captured are the mean SNR of each frame, and the $K$-factor of the wireless channel. The mean SNR is estimated taking as a reference the noise variance estimated during the silence preceding each frame; and the K-factor is estimated and averaged for all frames. Regarding simulations, the estimated mean SNRs for each frame in the real scenario are applied to the simulated transmissions, all with the same average $K$-factor. Finally, full characterization of the channel frequency responses or spatial covariance matrices have not been obtained, so simplified assumptions have been made while conducting the simulations. Basically, we assumed a frequency-flat spatially uncorrelated MIMO channel. The frequency-flat

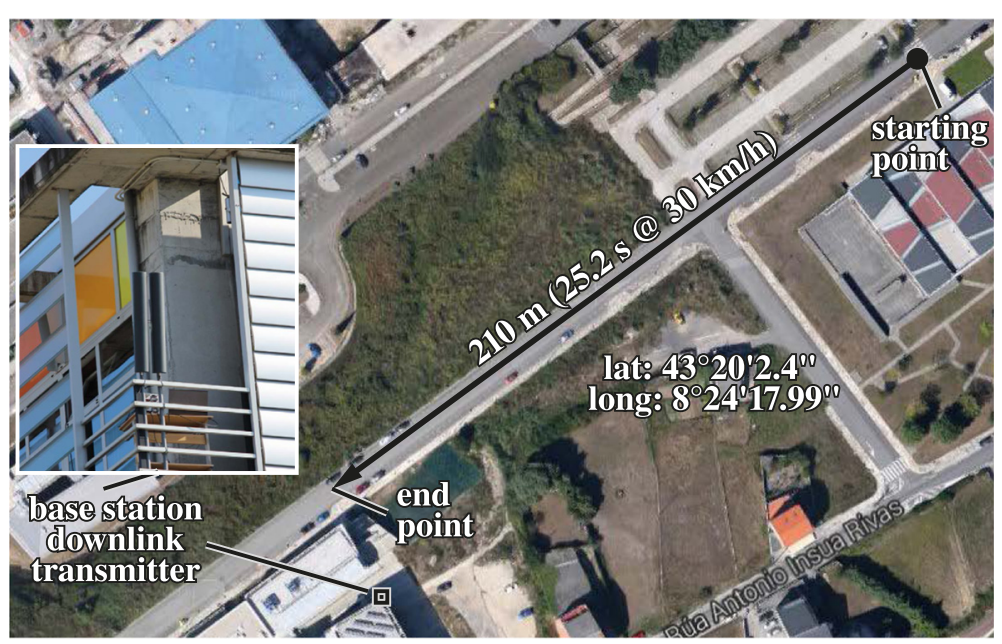

Figure 5 Measurement scenario at the Campus de Elviña, A Coruña. The measurement trajectory as well as the location of the base station are specified. 


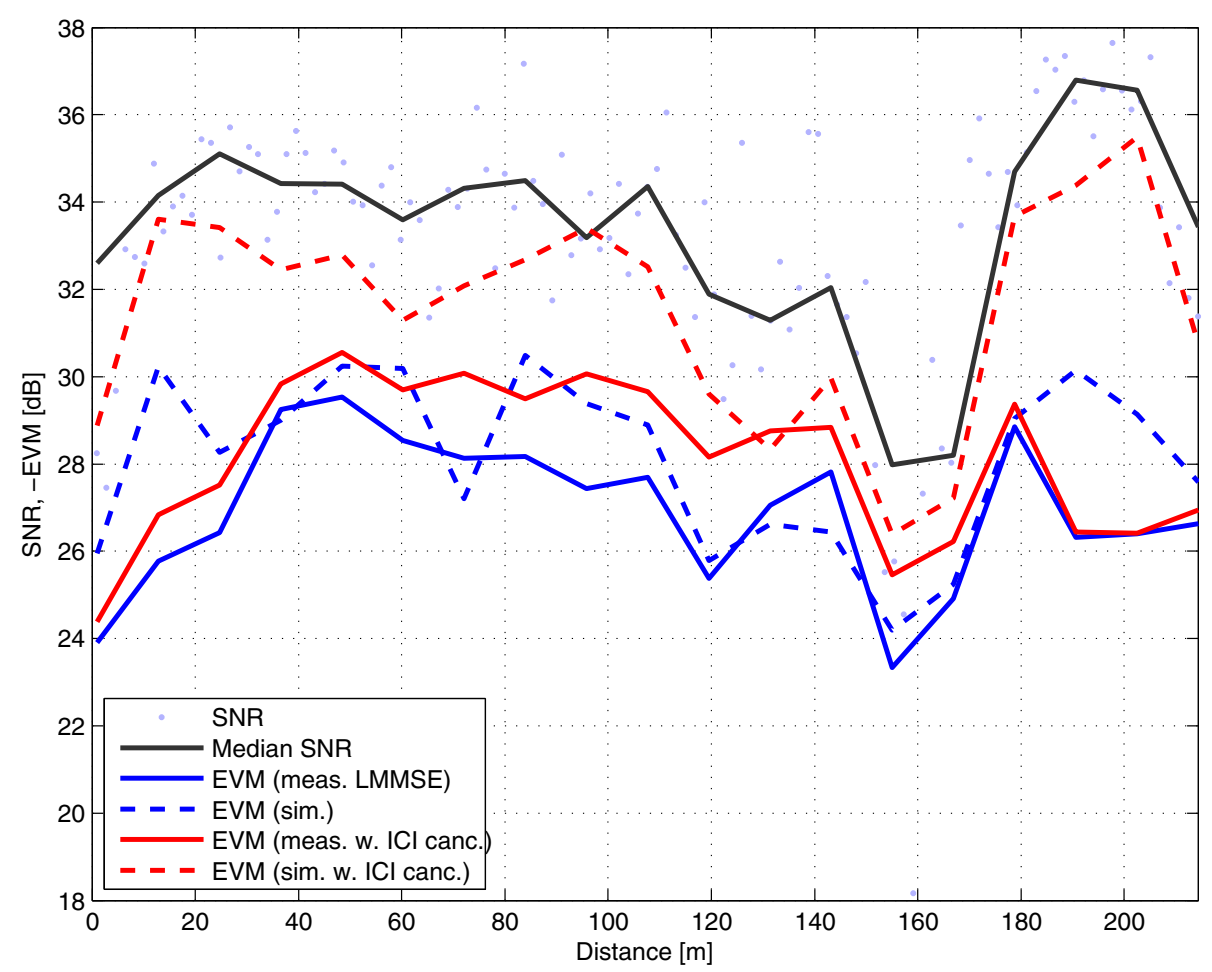

Figure $6 \mathrm{EVM}$ and SNR for $1 \times 1$ and $v^{\prime}=120 \mathrm{~km} / \mathrm{h}$. The receive antennas are placed outside the car.

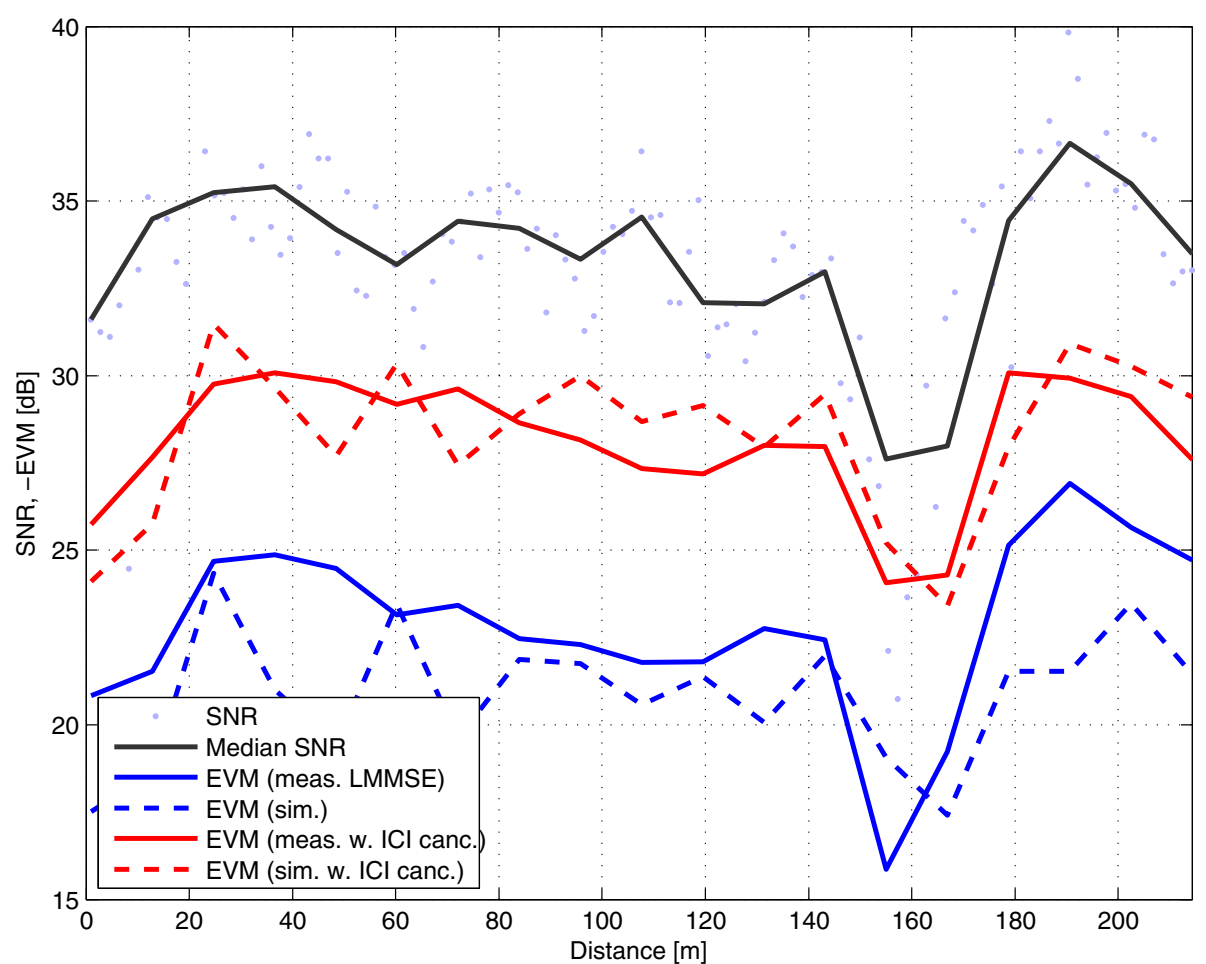

Figure 7 EVM and SNR for $1 \times 1$ and $v^{\prime}=360 \mathrm{~km} / \mathrm{h}$. The receive antennas are placed outside the car. 


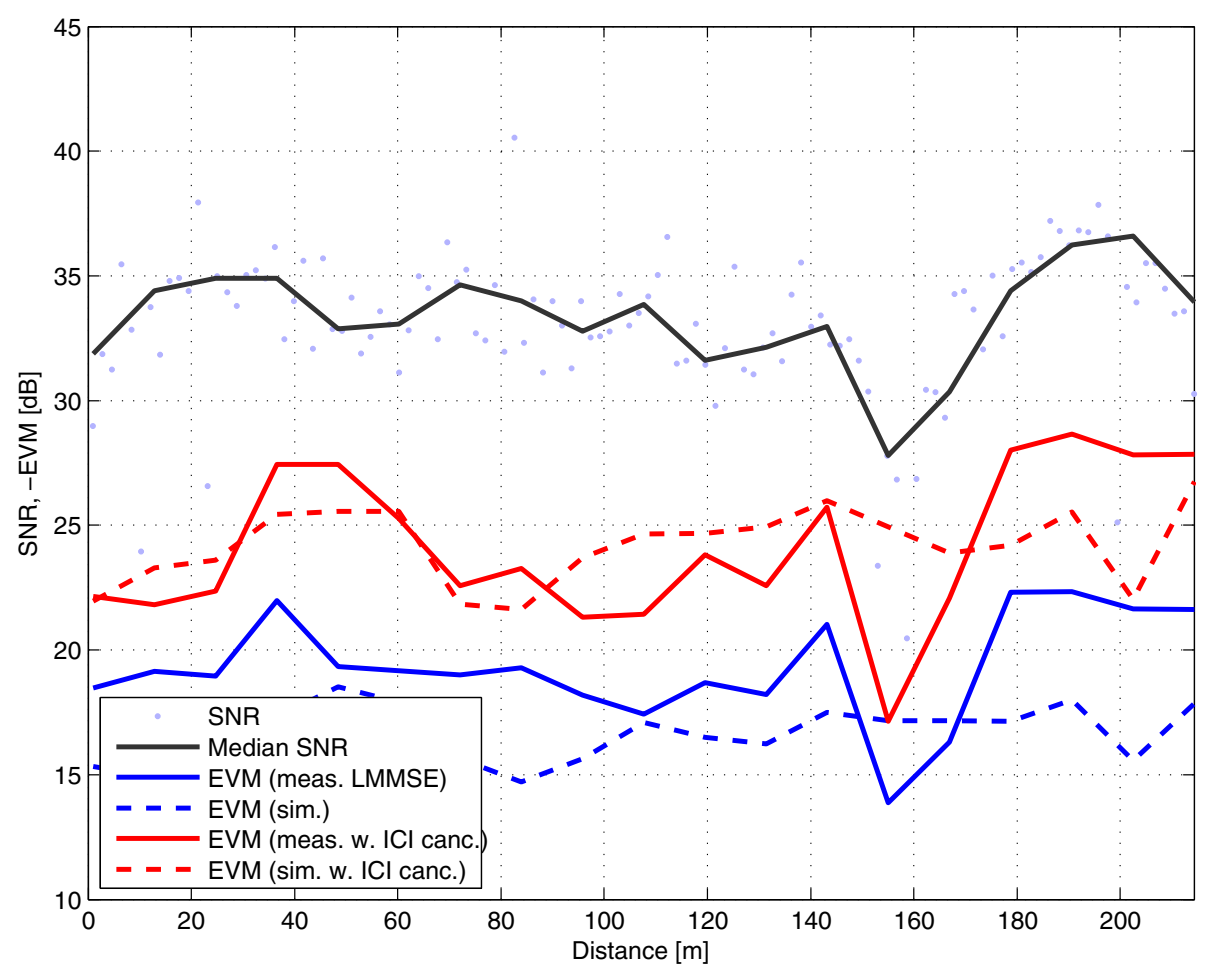

Figure $8 \mathrm{EVM}$ and SNR for $1 \times 1$ and $v^{\prime}=600 \mathrm{~km} / \mathrm{h}$. The receive antennas are placed outside the car.

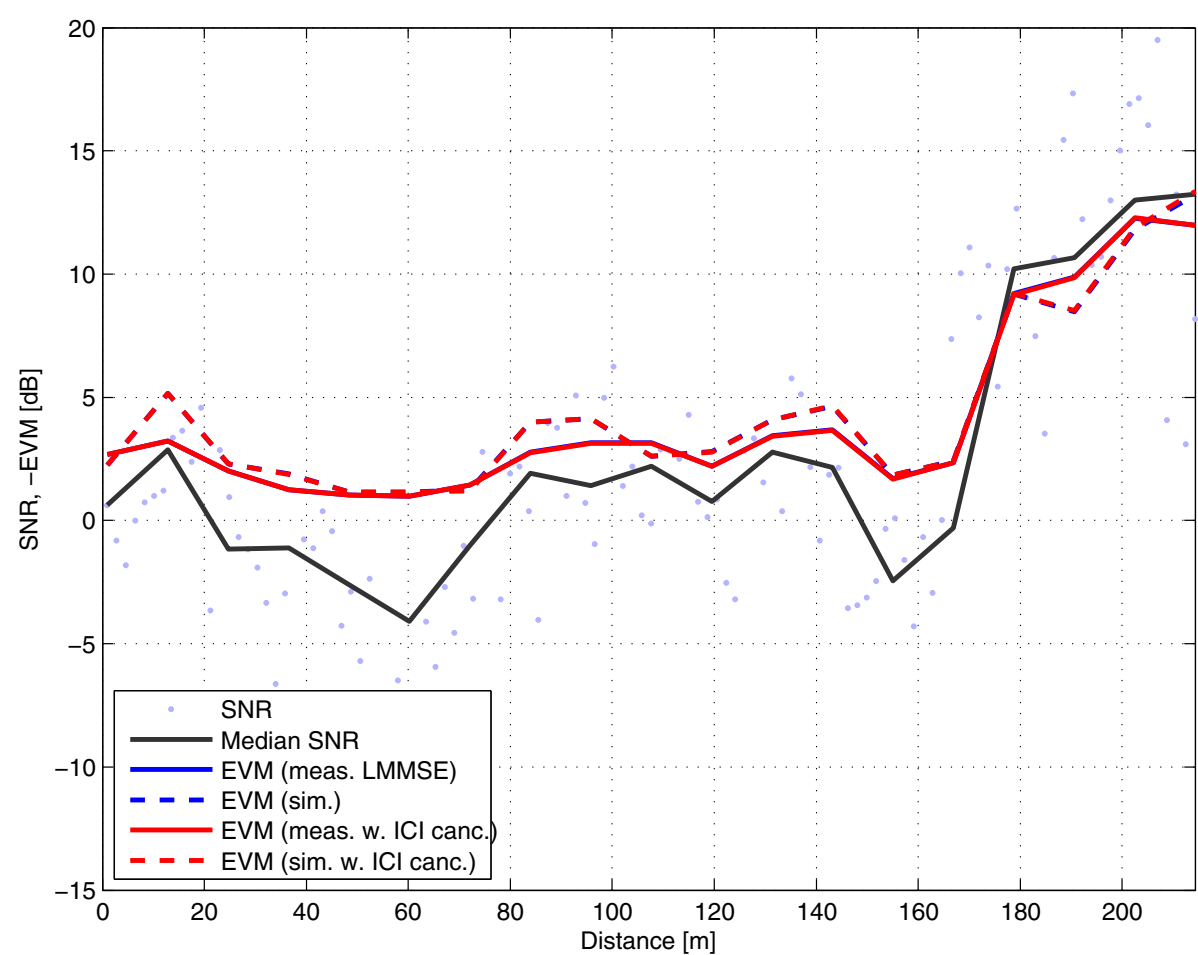

Figure $9 \mathrm{EVM}$ and SNR for $1 \times 1$ and $v^{\prime}=120 \mathrm{~km} / \mathrm{h}$. The receive antennas are placed inside the car. 


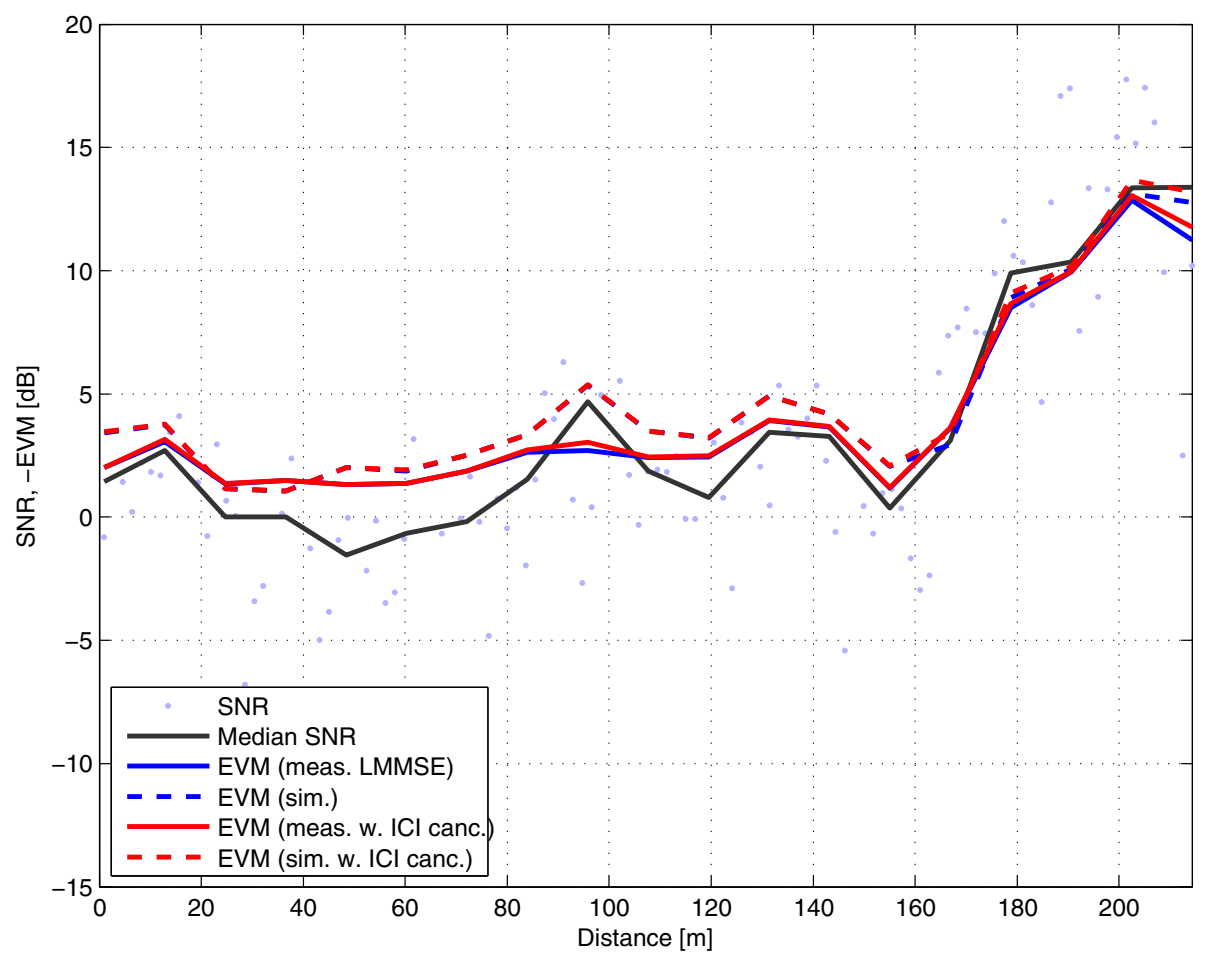

Figure $10 \mathrm{EVM}$ and SNR for $1 \times 1$ and $v^{\prime}=360 \mathrm{~km} / \mathrm{h}$. The receive antennas are placed inside the car.

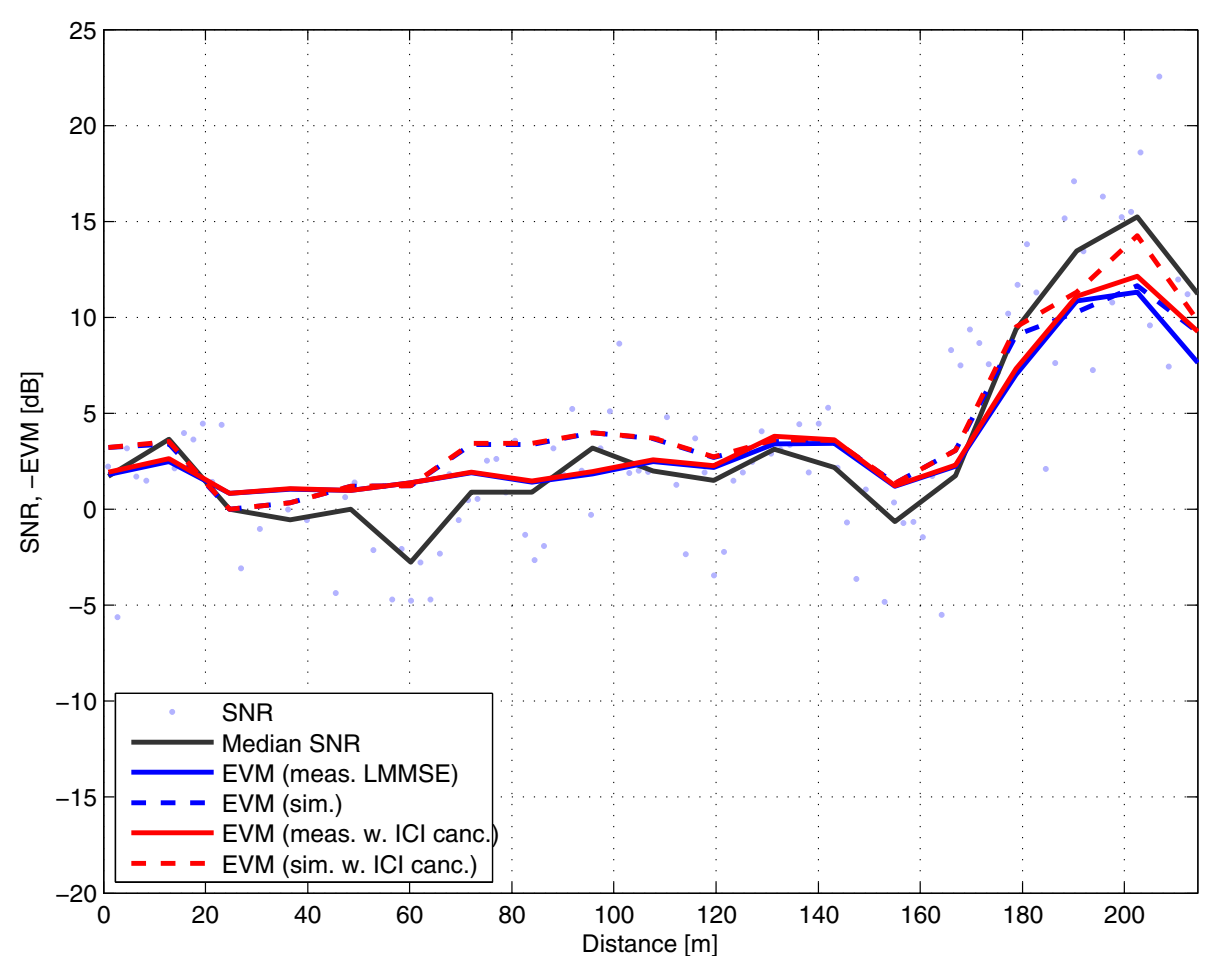

Figure 11 EVM and SNR for $1 \times 1$ and $v^{\prime}=600 \mathrm{~km} / \mathrm{h}$. The receive antennas are placed inside the car. 


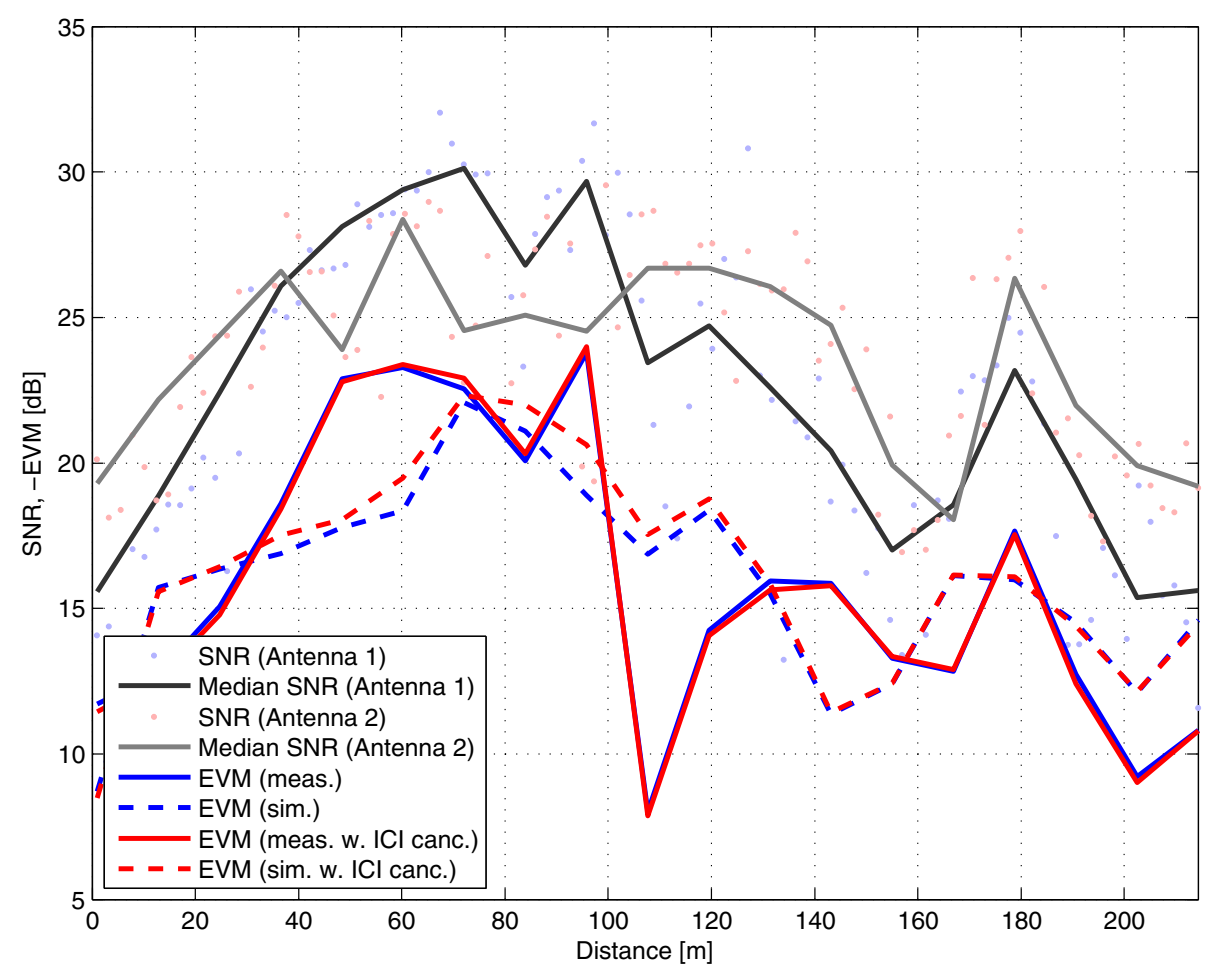

Figure 12 EVM and SNR for $2 \times 2$ and $v^{\prime}=120 \mathrm{~km} / \mathrm{h}$. The receive antennas are placed outside the car.

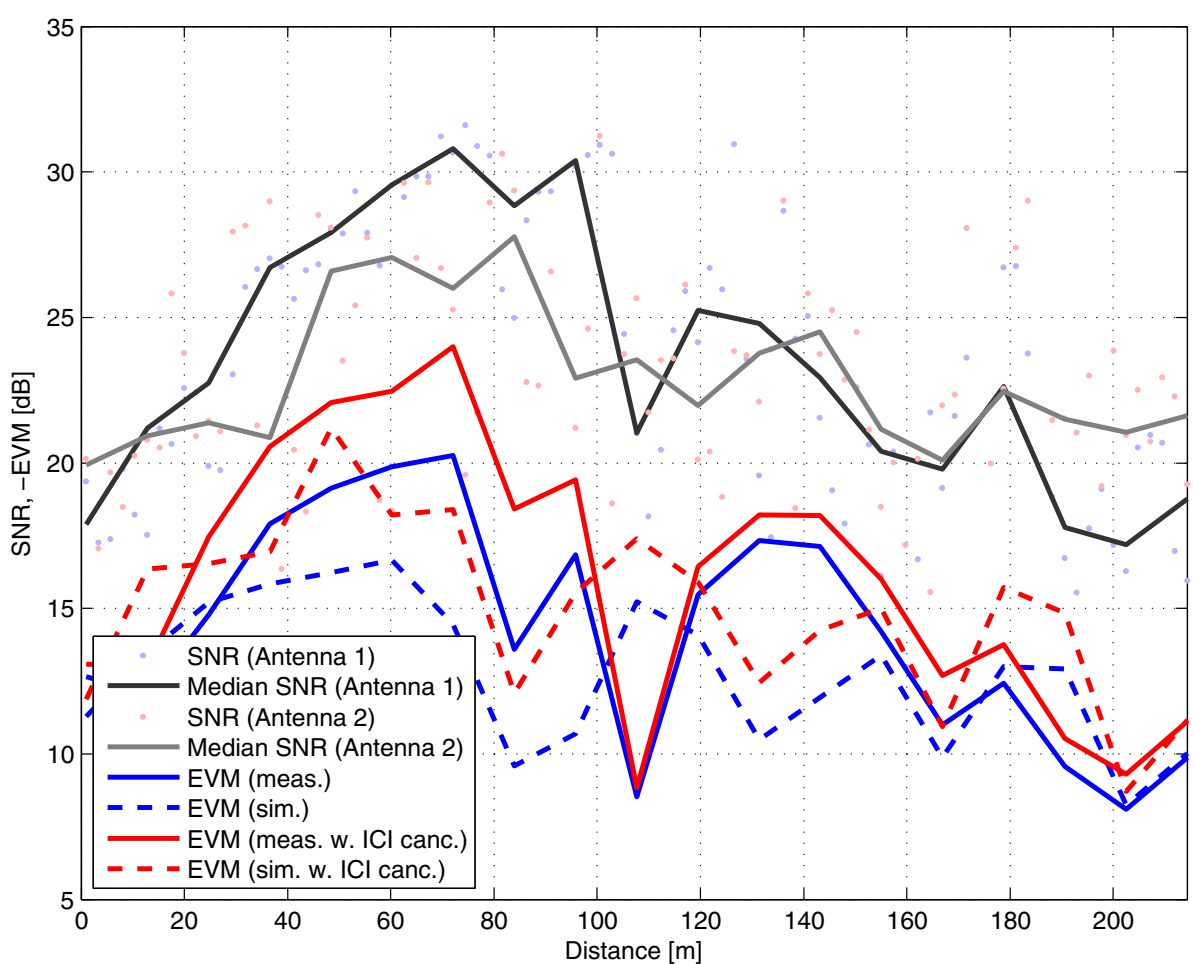

Figure $13 \mathrm{EVM}$ and SNR for $2 \times 2$ and $v^{\prime}=360 \mathrm{~km} / \mathrm{h}$. The receive antennas are placed outside the car. 


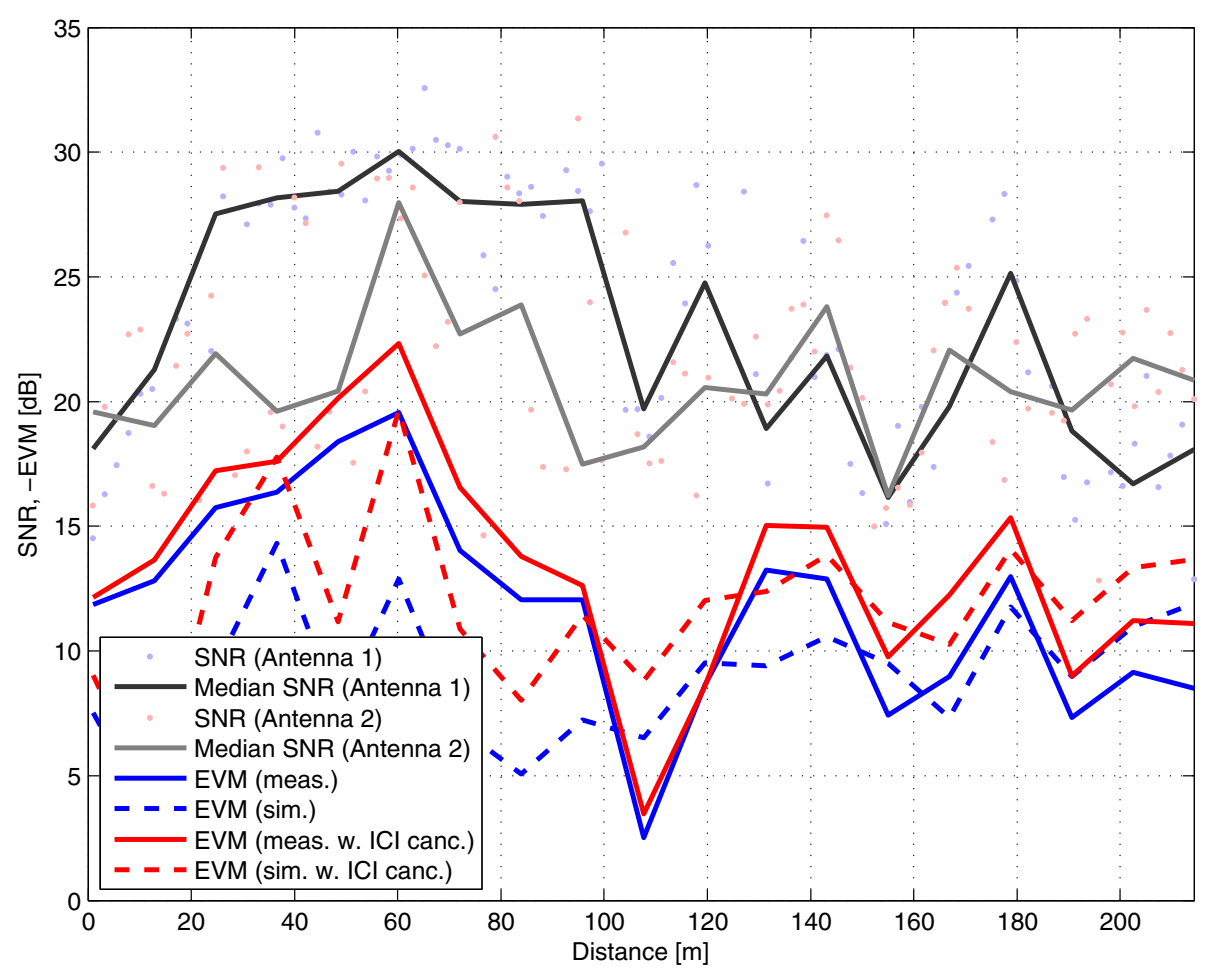

Figure 14 EVM and SNR for $2 \times 2$ and $v^{\prime}=600 \mathrm{~km} / \mathrm{h}$. The receive antennas are placed outside the car

assumption arises from the fact that for the highest interpolation factors, the bandwidth of the transmitted signals will be rather narrow, and therefore the frequency selectivity observed by the receiver would be negligible. As for the MIMO channel, the coefficients for each pair of antennas are drawn from independent Rice distributions, although not identically distributed, picking random complex mean values for each MIMO channel matrix entry.

\section{Error vector magnitude and SNR}

Error vector magnitude (EVM) is the first figure of merit considered in this work. EVM is measured as the mean squared error between the received constellation after equalization and the transmitted constellation, normalized by the transmitted constellation power, i.e.:

$$
\operatorname{EVM}_{F}=\frac{\sum_{k=1}^{K} \sum_{n=1}^{N}\left\|\hat{\mathbf{s}}_{k}(n)-\mathbf{s}_{k}(n)\right\|^{2}}{\sum_{k=1}^{K} \sum_{n=1}^{N}\left\|\mathbf{s}_{k}(n)\right\|^{2}},
$$

being $\mathrm{EVM}_{F}$ the mean EVM for the $F$ th frame. Note that the original transmitted symbols are used to normalize the (EVM). Notice that if only symbol estimates were available, EVM measures would be less accurate.

Figures 6, 7, and 8 show the EVM and SNR results versus distance for SISO transmissions with outdoor antennas for interpolation factors $I=4, I=12$, and $I=20$, respectively. The car moved at the actual speed of $30 \mathrm{~km} / \mathrm{h}$. The previous factors correspond to emulated speeds of 120 , 360 , and $600 \mathrm{~km} / \mathrm{h}$, respectively. To clarify the plots, the course of the car has been split in 20 equally long sectors, and the median EVM is determined for each sector. Also, as a reference, the median SNR for each sector is shown. Figures 6,7 , and 8 clearly show the performance gains obtained when ICI cancellation is performed at reception. The figures also show that comparable gains are obtained with simulation and real measurements, hence validating the performance of the ICI estimation and cancellation techniques utilized. Figure 6 shows a constant offset between simulations and measurements. Recall that

Table 2 EVM to burst-profile mapping for throughput estimations

\begin{tabular}{lc}
\hline Profile & -EVM [dB] \\
\hline $4-Q A M ~ 1 / 2$ & 5 \\
4-QAM 3/4 & 8 \\
16-QAM 1/2 & 10.5 \\
16-QAM 3/4 & 14 \\
64-QAM 1/2 & 16 \\
64-QAM 3/4 & 20
\end{tabular}




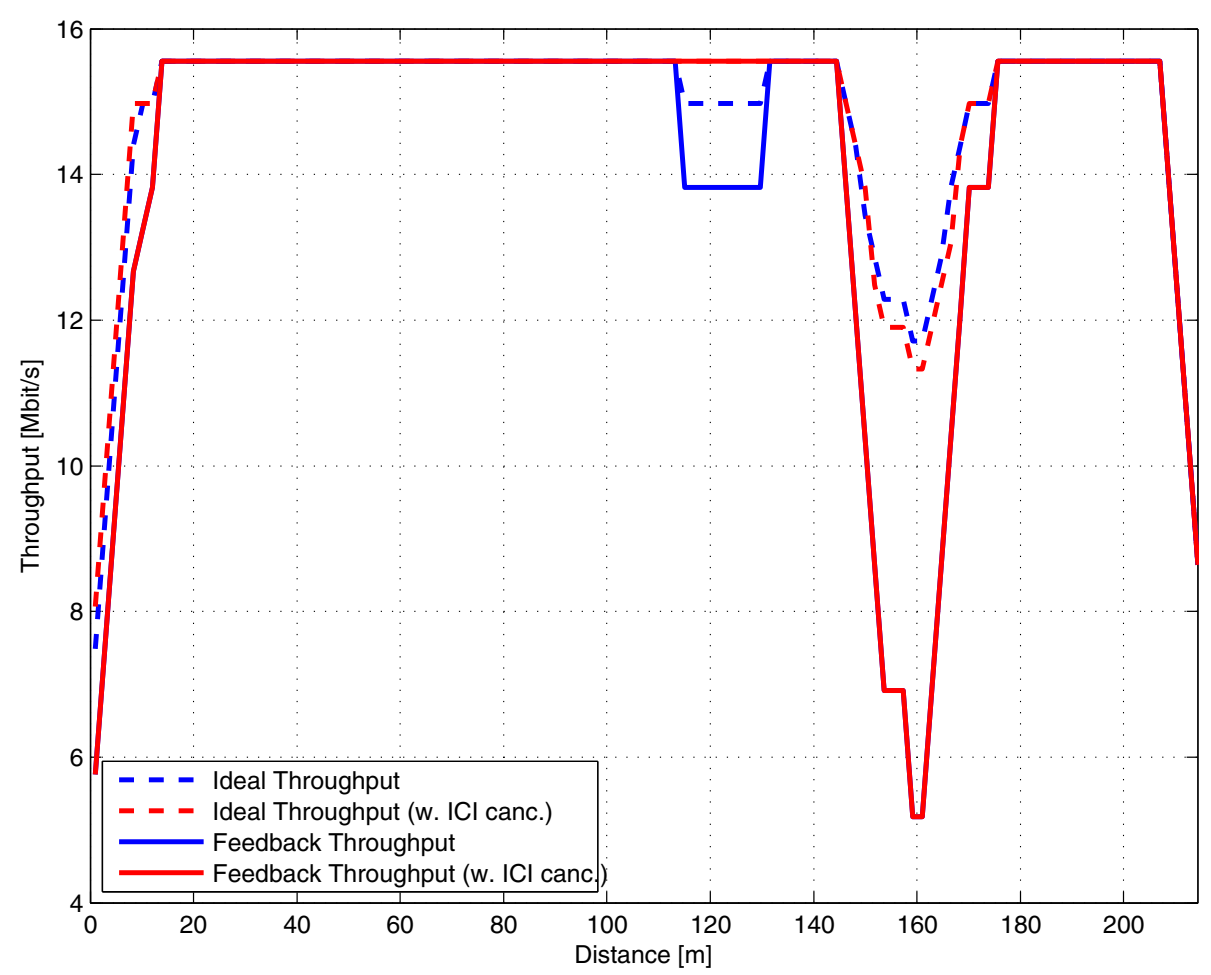

Figure 15 Throughput for $1 \times 1$ and $v^{\prime}=120 \mathrm{~km} / \mathrm{h}$. The receive antennas are placed outside the car.

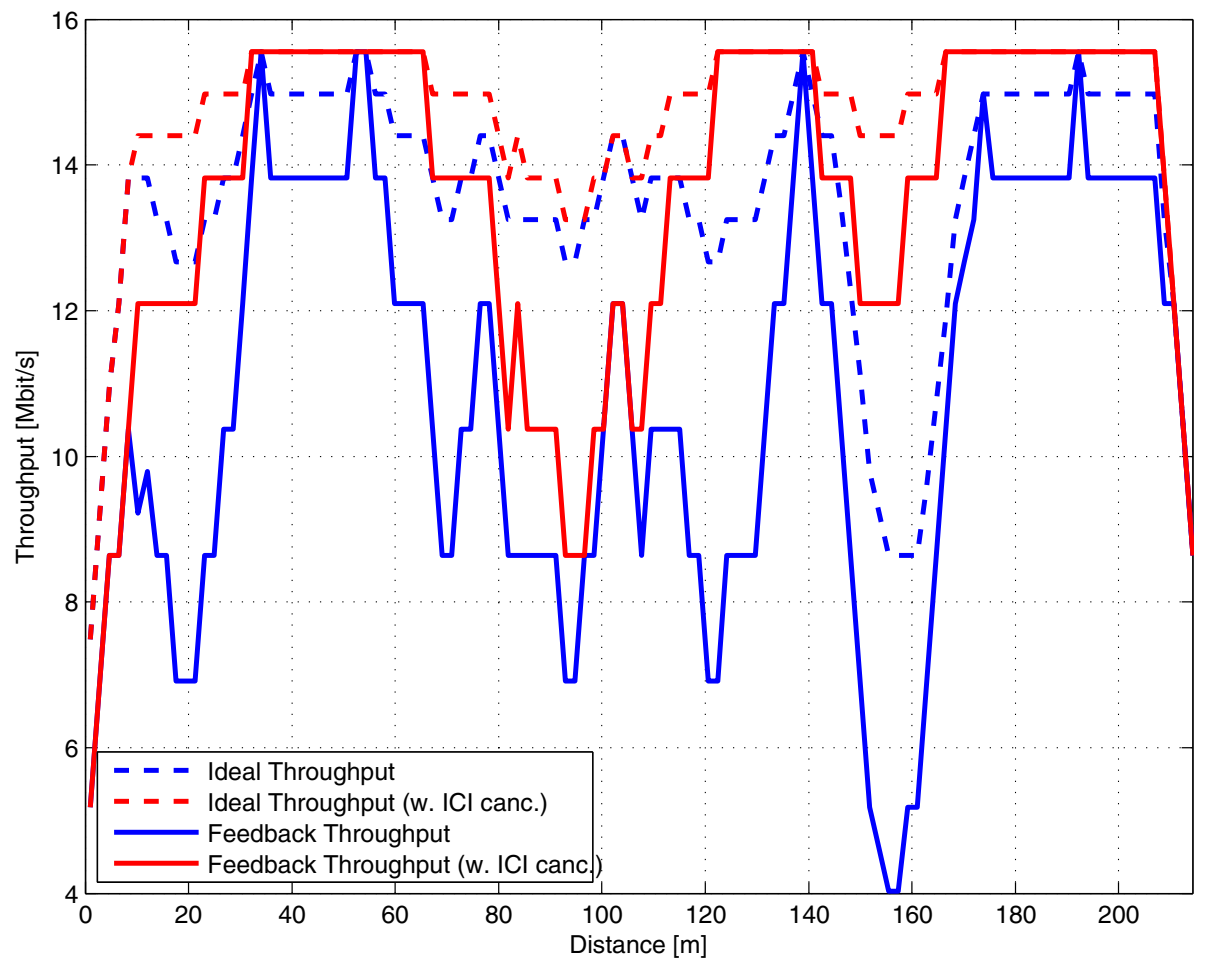

Figure 16 Throughput for $1 \times 1$ and $v^{\prime}=360 \mathrm{~km} / \mathrm{h}$. The receive antennas are placed outside the car. 


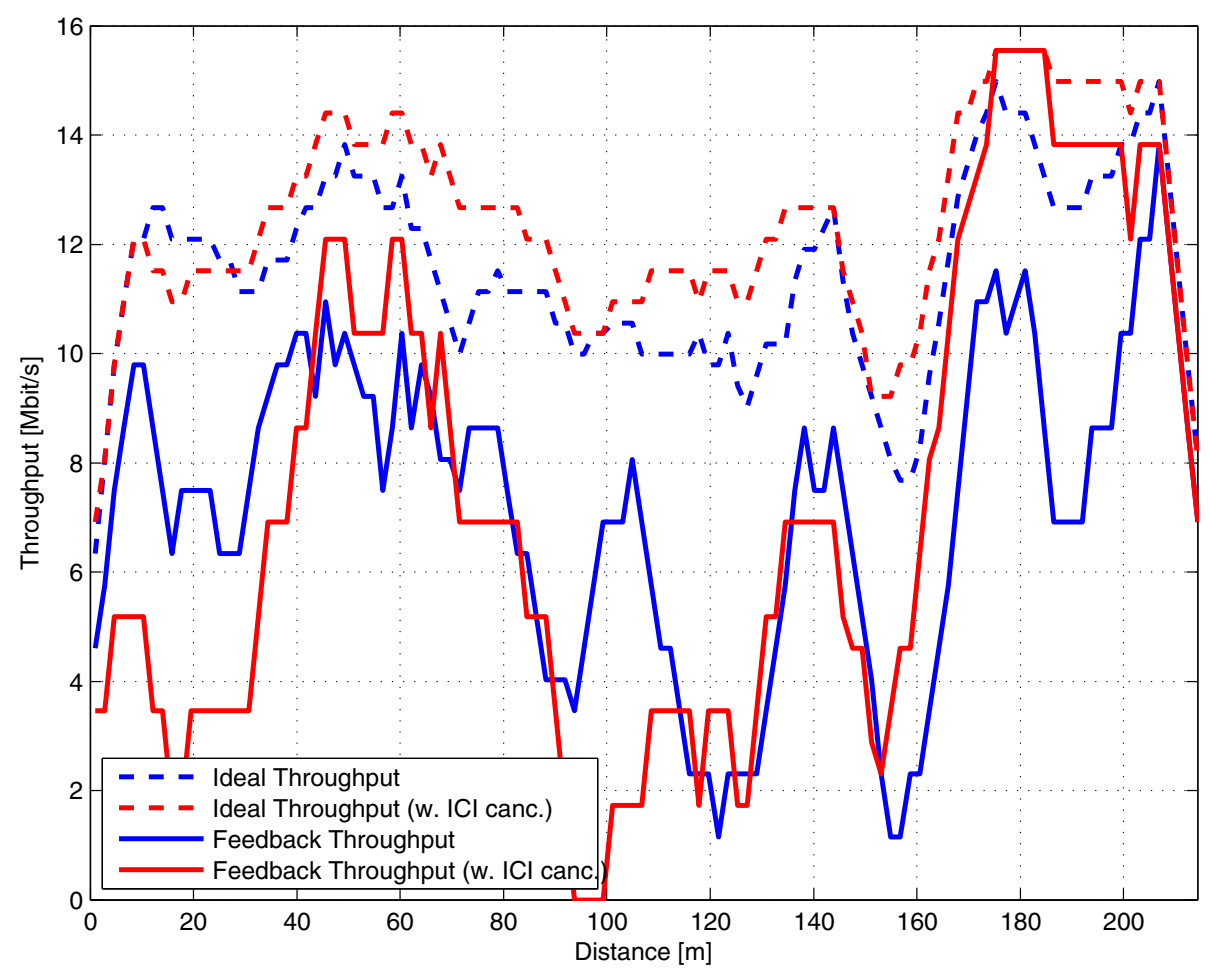

Figure 17 Throughput for $1 \times 1$ and $v^{\prime}=600 \mathrm{~km} / \mathrm{h}$. The receive antennas are placed outside the car.

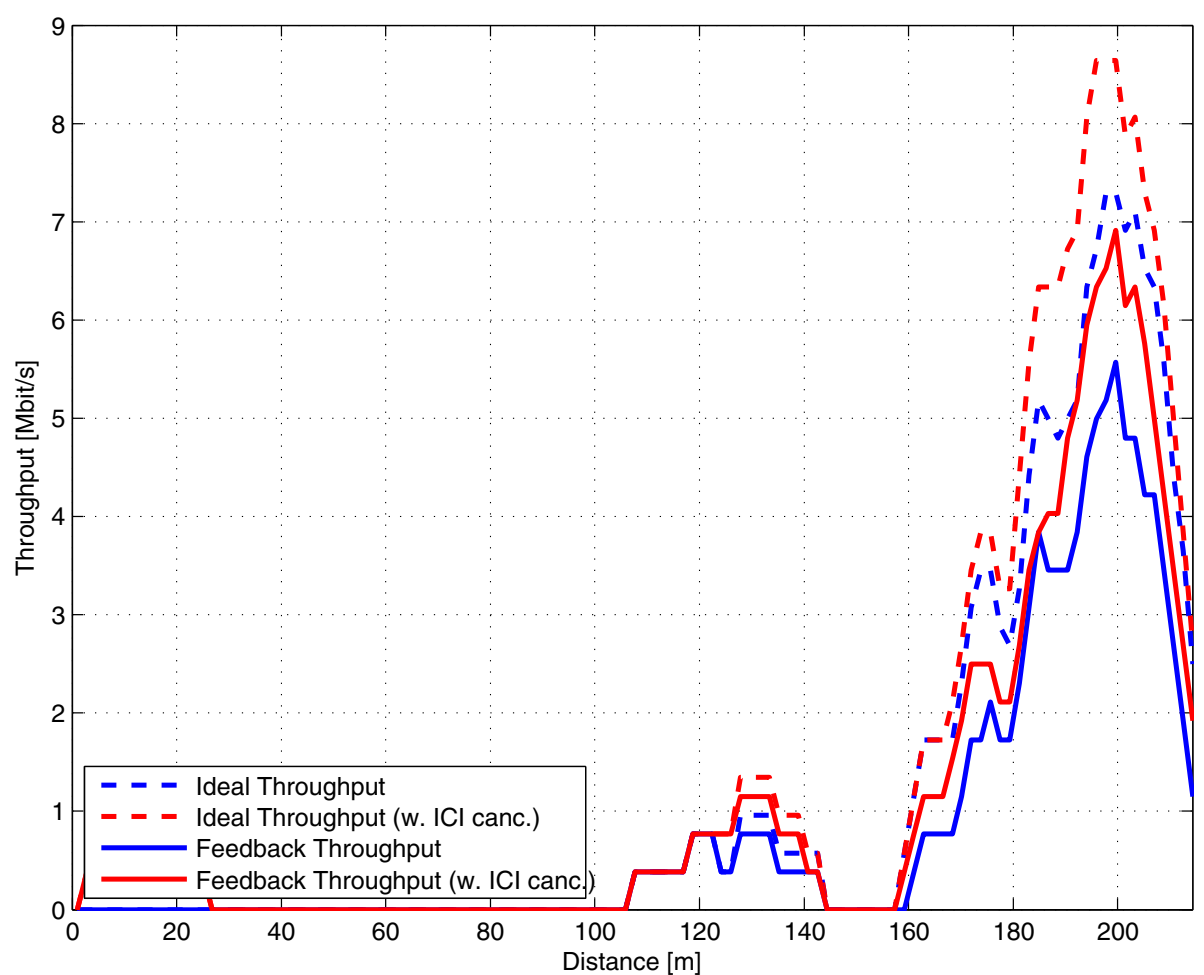

Figure 18 Throughput for $1 \times 1$ and $v^{\prime}=120 \mathrm{~km} / \mathrm{h}$. The receive antennas are placed inside the car. 


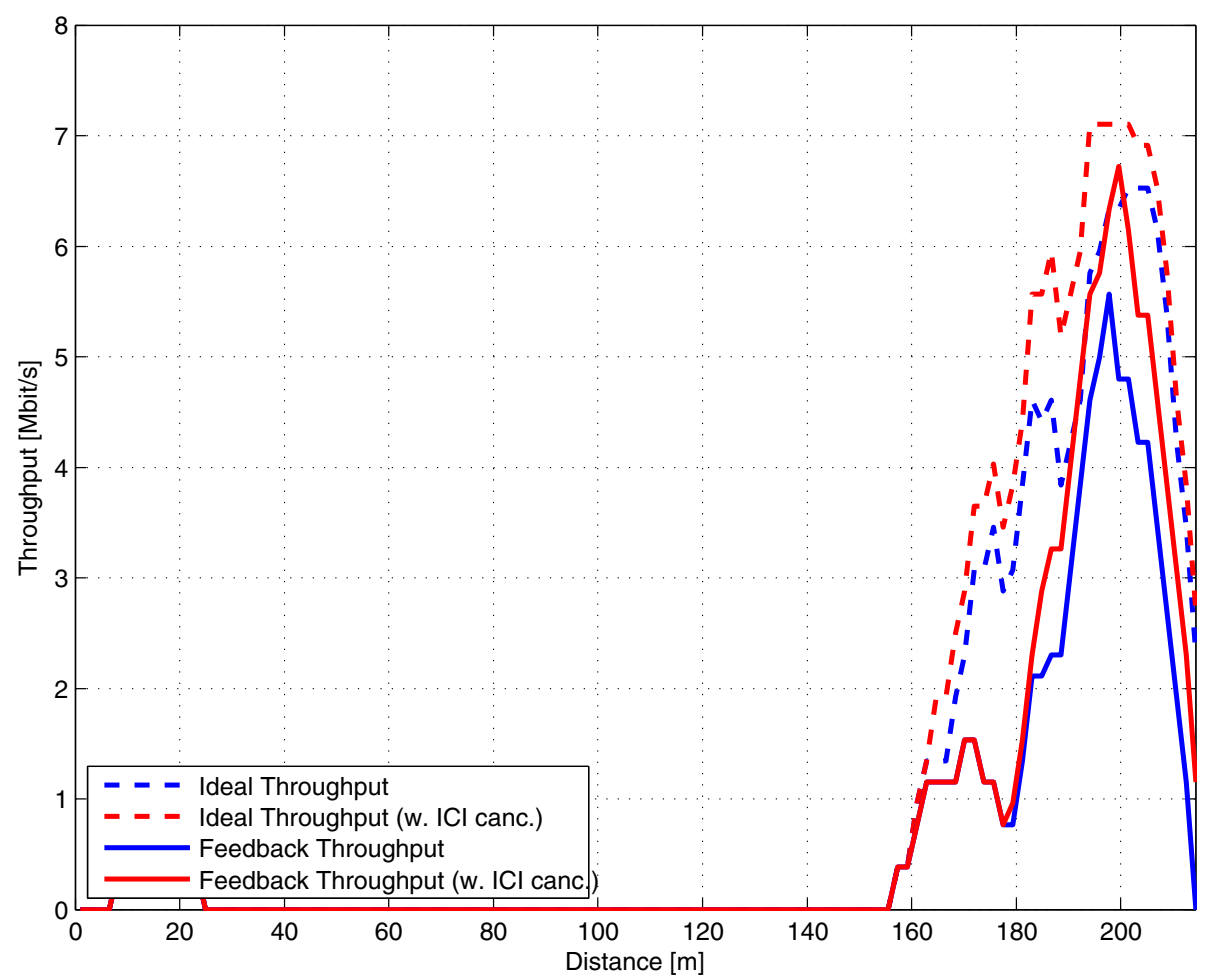

Figure 19 Throughput for $1 \times 1$ and $v^{\prime}=360 \mathrm{~km} / \mathrm{h}$. The receive antennas are placed inside the car.

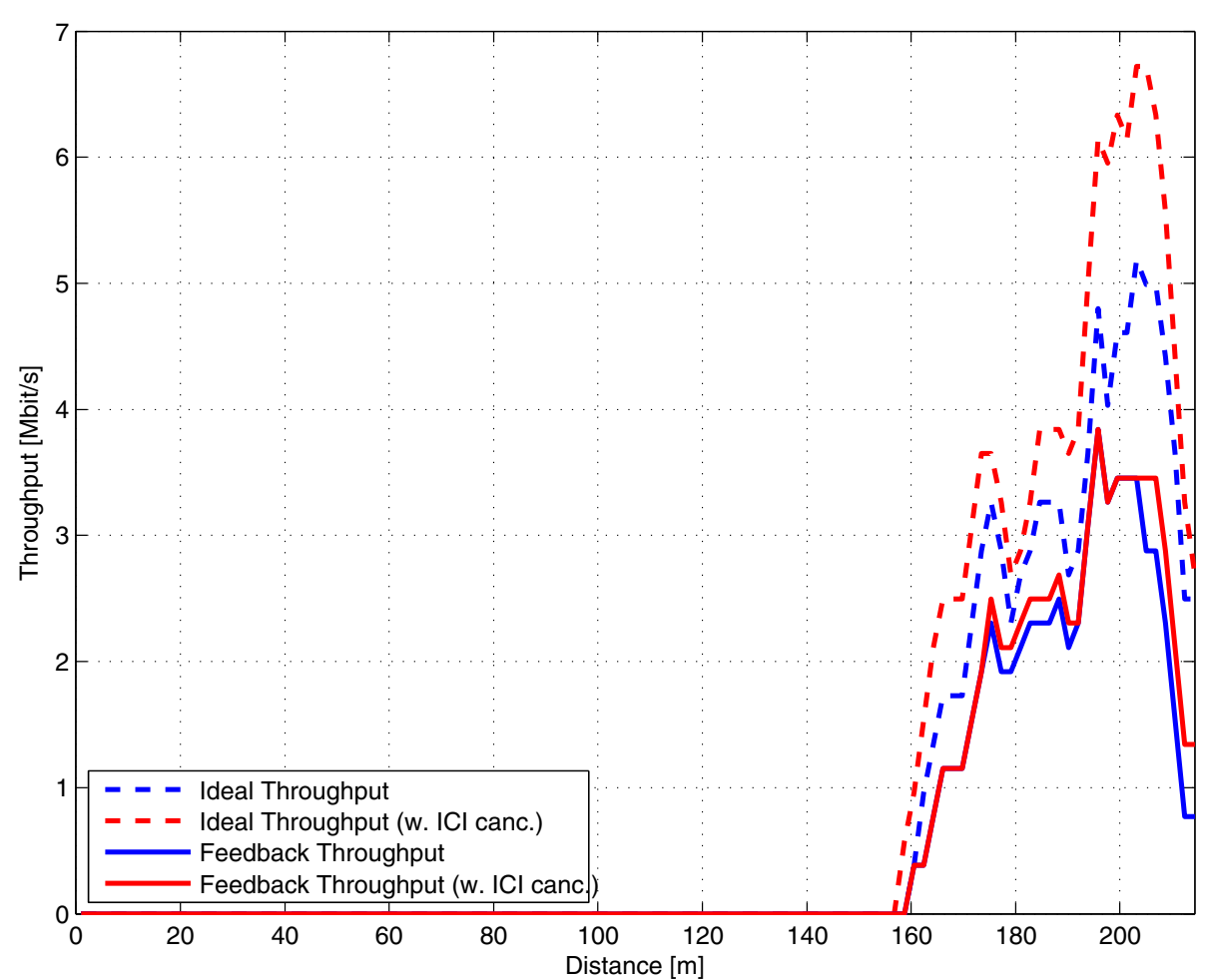

Figure 20 Throughput for $1 \times 1$ and $v^{\prime}=600 \mathrm{~km} / \mathrm{h}$. The receive antennas are placed inside the car. 


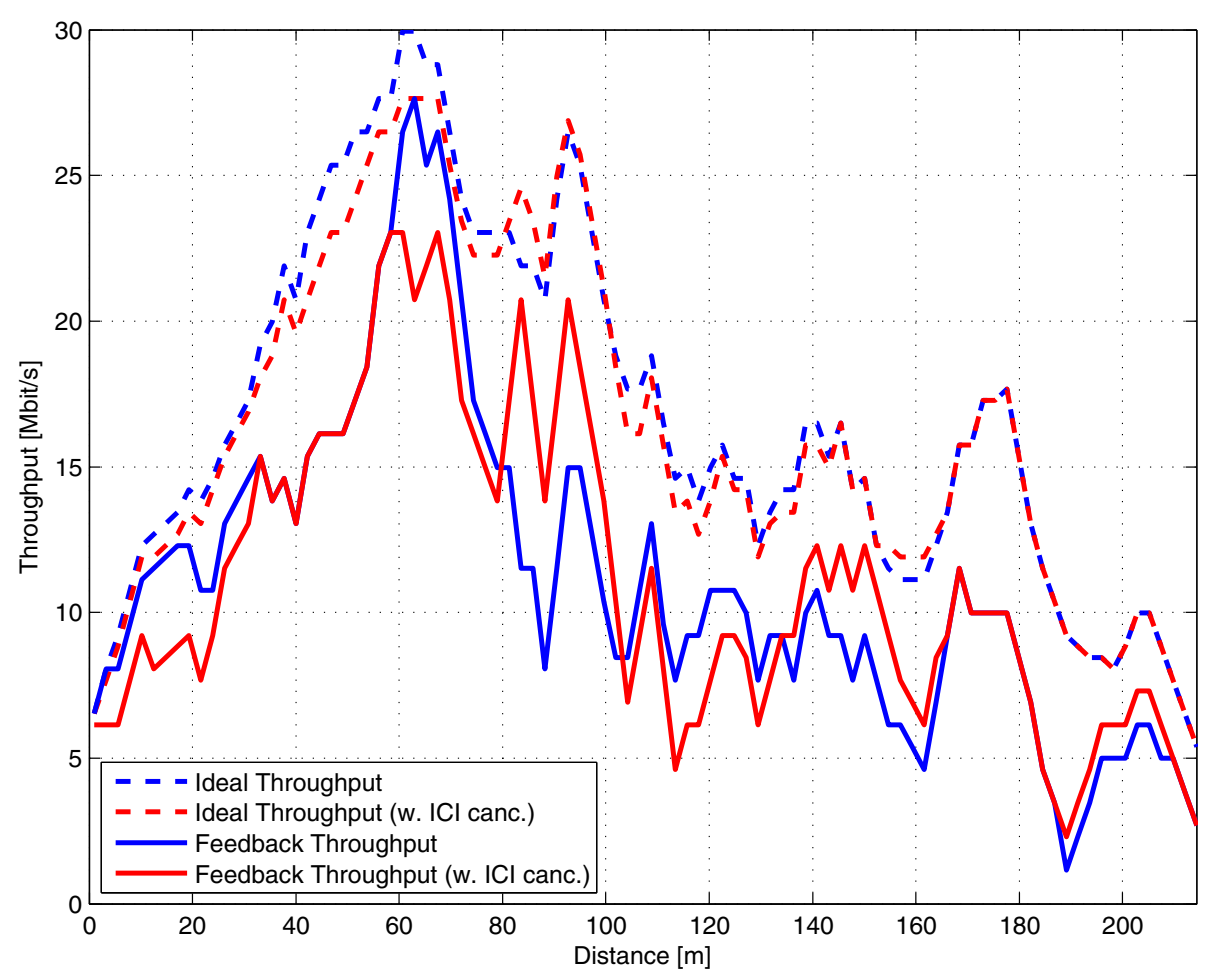

Figure 21 Throughput for $2 \times 2$ and $v^{\prime}=120 \mathrm{~km} / \mathrm{h}$. The receive antennas are placed outside the car.

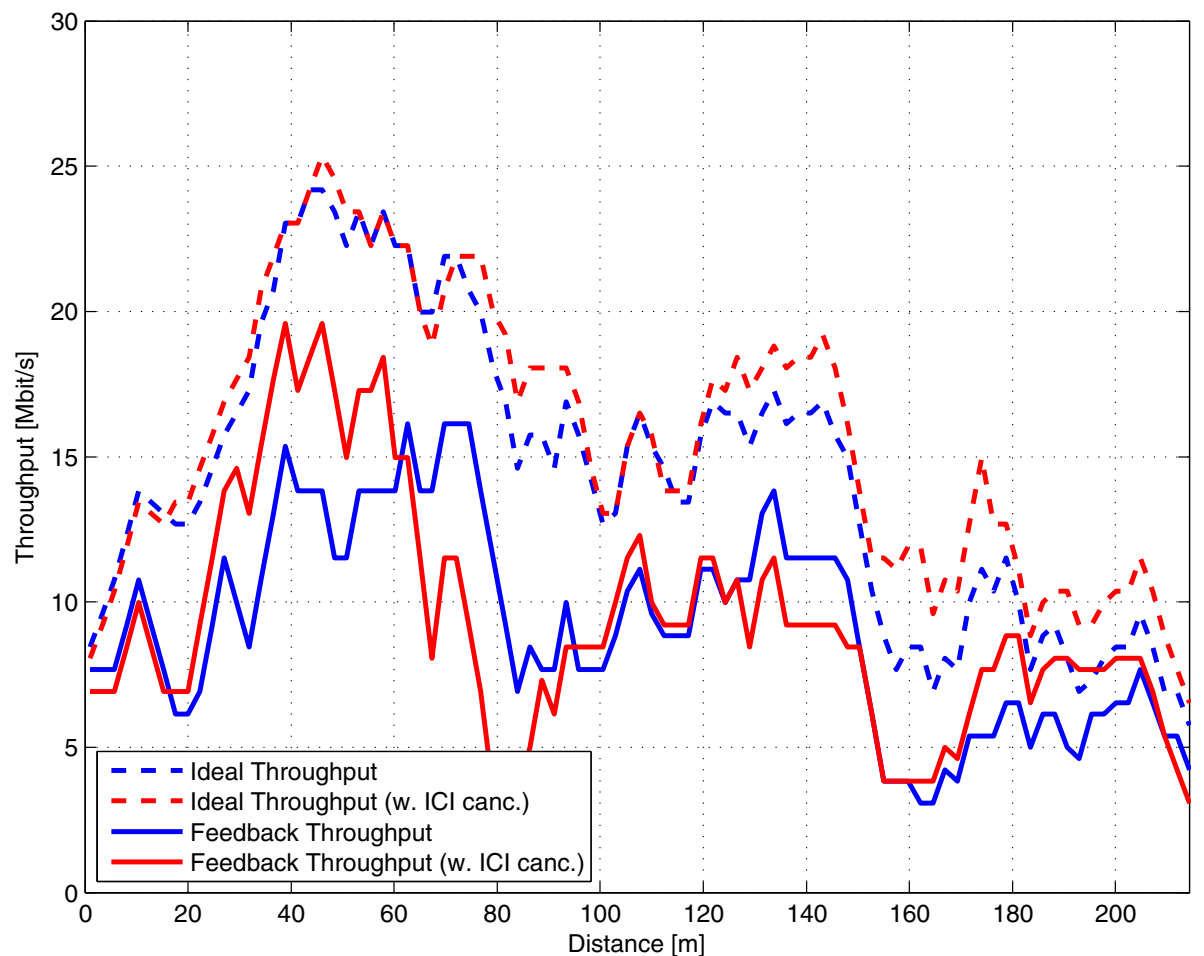

Figure 22 Throughput for $2 \times 2$ and $v^{\prime}=360 \mathrm{~km} / \mathrm{h}$. The receive antennas are placed outside the car. 


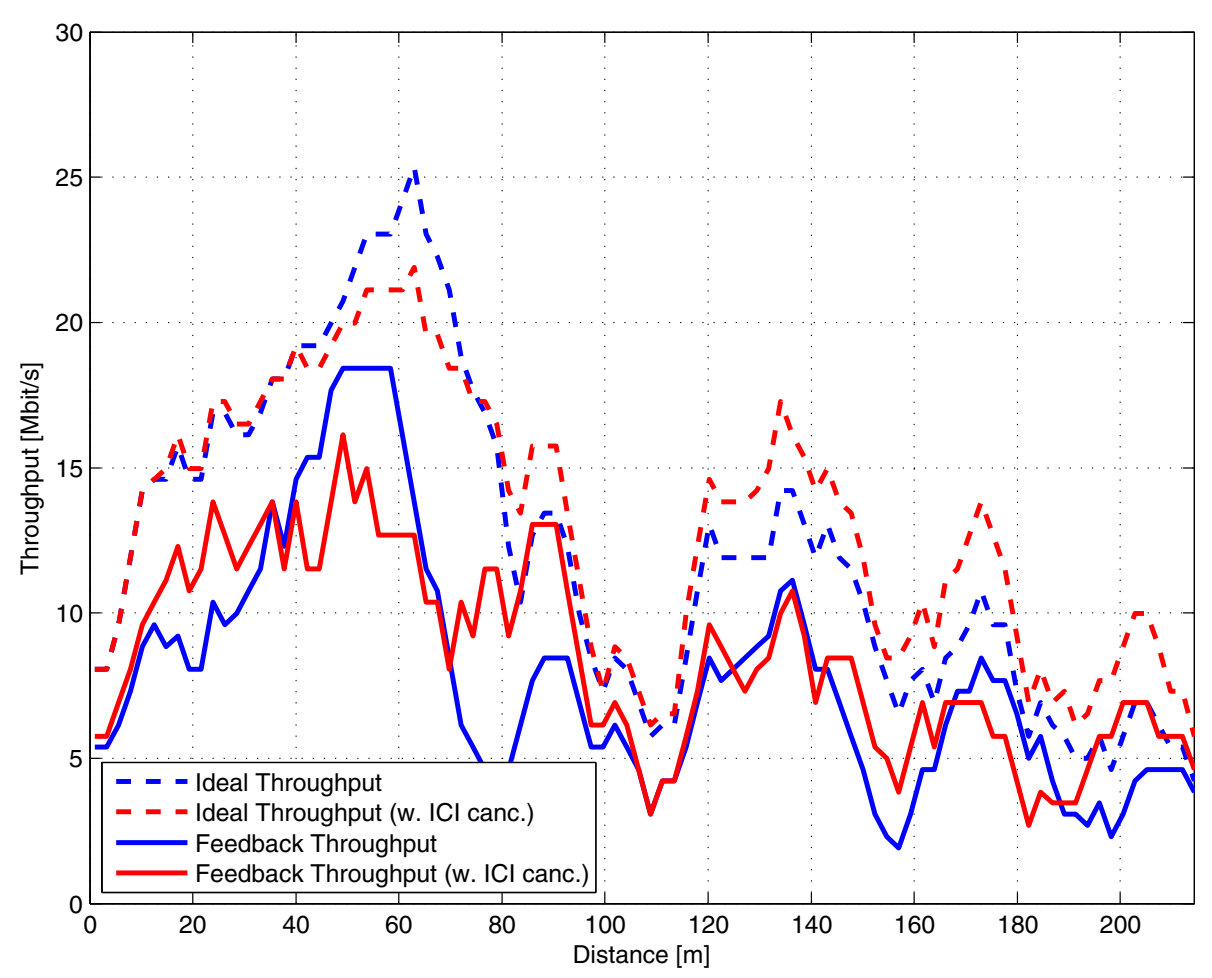

Figure 23 Throughput for $2 \times 2$ and $v^{\prime}=600 \mathrm{~km} / \mathrm{h}$. The receive antennas are placed outside the car.

simulation results were obtained assuming a frequencyflat channel. The soundness of this assumption weakens for low interpolation factors such as $I=4$, so a significant difference between simulation and experimental results is to be expected. Conversely, better agreement between experiments and simulations is observed in Figures 7 and 8 which correspond to higher interpolation factors $(I=12$ and $I=20$, respectively). In such cases, the frequency-flat channel response assumption is tighter.

Figures 9, 10, and 11 show the results for the same measurements as before but with the mobile receiver inside the car. The most important difference compared to the previous figures is the lower SNR of this scenario, where median values range between 0 and $10 \mathrm{dBs}$, a large difference compared to the 30 to $35 \mathrm{~dB}$ achieved outside due to the strong attenuation introduced by the car structure. As a consequence, negligible performance gains are obtained with receivers that perform ICI cancellation because the noise at the receiver prevails over the interference of the neighbor subcarriers.

Figures 12,13, and 14 show the results corresponding to $2 \times 2$ MIMO measurements with the receiving antennas placed outside the car for $I=4, I=12$, and $I=$ 20 , respectively. Contrary to the SISO case, ICI cancellation does not produce significant performance gains when $I=4$. ICI cancellation produces significant performance gains for $I=12$ and $I=20$. Again, this is because the received SNR is significantly smaller and the Gaussian noise dominates over the ICI. Also, although spatially uncorrelated channels have been assumed in simulations, the real scenario will show some spatial correlation between antennas. Such correlation depends on the distance between antennas, and also on the distance between the transmitter and the receiver, an effect more evident when strong line-of-sight components are present [30]. This spatial correlation introduces interference between

Table 3 Mean throughput in Mbit/s for the $1 \times 1$ scenario when the receive antennas are placed outside the car

\begin{tabular}{lcccc}
\hline & Ideal throughput & $\begin{array}{c}\text { Ideal throughput } \\
\text { (with ICI cancellation) }\end{array}$ & $\begin{array}{c}\text { Feedback throughput } \\
\text { I }\end{array}$ & $\begin{array}{r}\text { Feedback throughput } \\
\text { (with ICI cancellation) }\end{array}$ \\
$I=4$ & 14.32 & 14.73 & 13.01 & 13.93 \\
$I=20$ & 12.22 & 13.78 & 8.81 & 11.39 \\
\hline
\end{tabular}


Table 4 Mean throughput in Mbit/s for the $1 \times 1$ scenario when the receive antenna is placed inside the car

\begin{tabular}{lcccr}
\hline & Ideal throughput & $\begin{array}{c}\text { Ideal throughput } \\
\text { (with ICl cancellation) }\end{array}$ & $\begin{array}{c}\text { Feedback throughput } \\
\begin{array}{r}\text { Feedback throughput } \\
\text { (with ICl cancellation) }\end{array}\end{array}$ & 2.45 \\
$I=4$ & 3.15 & 3.48 & 2.11 & 2.23 \\
$I=20$ & 2.90 & 3.26 & 1.96 & 1.97 \\
\hline
\end{tabular}

antennas, which impairs the gain that could be obtained by the ICI cancellation technique.

\section{Throughput}

In order to evaluate the impact of the ICI cancellation methods on the final user quality of experience (QoE), throughput estimations have been carried out using the information conveyed in the bursts. Two types of throughput estimation have been considered. The first one assumes that the transmitter knows, for each frame, the optimal modulation and coding profile to be used. This case was modeled by estimating the bit error ratio (BER) of the six received bursts per frame and assuming that the whole frame was transmitted with the profile which carries more information bits without suffering any bit error. The second method assumes that the transmitter estimates the most suitable burst profile, and consequently only transmits the corresponding profile during all the burst. Errors are measured in this case assuming only the burst picked by the transmitter is available, regardless whether this burst has errors or not. This method is intended to determine the impact of the channel fast time-variations on this type of decisions.

To allow the transmitter to take these decisions, a frame by frame EVM metric is obtained in a similar way as the WiMAX standard proposes, i.e.:

$$
\mathrm{EVM}_{F}=(1-\alpha) \mathrm{EVM}_{F-1}+\alpha \mathrm{EVM}_{F}
$$

where $\alpha$ is a forgetting factor. Along this work, we assume $\alpha=1 / 4$. This EVM value is used to pick a modulation profile and burst according to Table 2 .

Results for the $1 \times 1$ setup with the antennas placed outside the car are shown in Figures 15, 16, and 17 for interpolation factors $I=4, I=12$, and $I=20$, respectively. When $I=4$, the performance gain when using the receiver with ICI cancellation is not significant because the throughput already saturates at the maximum value
$15 \mathrm{Mbit} / \mathrm{s}$ without cancelling the ICI (see Figure 15). On the contrary, significant performance gains are obtained for $I=12$ and $I=20$ when the ICI is cancelled. Nevertheless, note the large throughput degradation when the profile is determined from delayed EVM measurements rather than from the actual ones. In channels with low coherence times, estimations of the EVM according to Equation 13 are less reliable, thus increasing the probability of picking up an incorrect burst profile. This effect is more evident for the highest interpolation factor $I=20$, where at some time instants even the ICI-cancelled signal provides lower throughput.

Figures 18, 19, and 20 show the results for the same measurement campaign as before but with the antennas placed inside the car. The most important effect is that the SNR is now much lower and the receiver is unable to detect any burst without errors during the first part of the measurement trajectory. The larger SNRs observed in the last sector of the measurement course allow for correctly decoding information bits but leading to throughput values far from those observed from the antennas placed outside the car. Also, the throughput gains obtained after ICI cancellation are quite low.

Corresponding results for the $2 \times 2$ MIMO setup are shown in Figures 21, 22, and 23. The first evident feature is the larger achievable throughput, since two streams are being transmitted in each frame. As with EVM, low gains when $I=4$ are appreciated, but peak gains of $2 \mathrm{Mbit} / \mathrm{s}$ are observed when $I=12$ and $I=20$.

A more meaningful way to represent the mean throughputs obtained during all measurement campaigns is the one shown in Tables 3, 4, and 5. These throughputs show that in general, some gain is obtained by using receivers that cancel the ICI and how the throughput worsens with the interpolation factor. It is also important to note that in some scenarios at low-emulated speeds, negligible gains, or even less performance is obtained with these methods.

Table 5 Mean throughput in Mbit/s for $2 \times 2$ scenario when the receive antennas are placed outside the car

\begin{tabular}{lcccc}
\hline & Ideal throughput & $\begin{array}{c}\text { Ideal throughput } \\
\text { (with ICl cancellation) }\end{array}$ & $\begin{array}{c}\text { Feedback throughput } \\
\text { I }\end{array}$ & $\begin{array}{r}\text { Feedback throughput } \\
\text { (with ICl cancellation) }\end{array}$ \\
$I=12$ & 16.95 & 17.46 & 10.56 & 10.99 \\
$I=20$ & 15.74 & 17.48 & 9.20 & 11.01 \\
\hline
\end{tabular}


In general, a remarkable difference is observed between the ideal throughput and the realistic measure which considers the EVM metric to pick the burst profile. This is due to the inability of the EVM estimator to track the fast time-variations of the channel. Also, even though in the previous figures at some instants the ICI-cancelled throughput was lower, it can be seen that in average the ICI cancellation techniques provide a better performance. Finally, in the case of MIMO $2 \times 2$, although in terms of EVM almost no difference is appreciated when $I=4$, in terms of throughput an average gain of approximately $0.50 \mathrm{Mbit} / \mathrm{s}$ has been obtained. And when $I=12$ and $I=20$, the average gains grow up to 1.8 and $1.4 \mathrm{Mbit} / \mathrm{s}$ approximately.

\section{Conclusions}

We have experimentally evaluated the performance of the WiMAX downlink physical layer in high-mobility scenarios. Both SISO and MIMO transmissions were considered, as well as placing the receive antennas outside and inside the car used for the experiments. We focused on the ICI caused by channel time variations in such scenarios. Costeffective measurement campaigns were carried out where high-mobility scenarios are emulated with a vehicle moving at a low speed. The key idea is the enlargement of the symbol period prior to its transmission over the air. Such enlargement reduces the frequency spacing between the OFDM subcarriers in WiMAX transmissions and hence induces high ICI values on the received signals. Experiments illustrate the performance of WiMAX receivers with and without ICI cancellation in terms of EVM and throughput. ICI cancellation produces significant performance gains mainly when the received SNR is high. Otherwise, thermal noise dominates over the ICI, and gains are not so much appreciated. Furthermore, the spacing of subcarriers in this standard is sufficient to provide a robust behavior for moderate mobility environments, and the gain observed after ICI cancellation is not very significant for speeds up to $80 \mathrm{~km} / \mathrm{h}$. Note that the profile tested in this work is the WiMAX profile with the lower subcarrier spacing, so it is foreseeable that other profiles present better performance under these scenarios.

\section{Competing interests}

The authors declare that they have no competing interests.

\section{Acknowledgements}

The authors thank Ismael Rozas Ramallal for his support in developing and testing the testbed and conducting the measurement campaigns. This work was supported in part by Xunta de Galicia, MINECO of Spain, and by FEDER funds of the E.U. under Grant 2012/287, Grant IPT-2011-1034-370000, and Grant TEC2013-47141-C4-1-R.

Received: 14 December 2014 Accepted: 24 March 2015 Published online: 18 April 2015

\section{References}

1. UQ WiMAX gaining the wireless broadband market (2013). http://www. wimaxforum.org/LiteratureRetrieve.aspx?|D=177978
2. M Ehammer, E Pschernig, T Graupl, in IEEE/AIAA 30th Digital Avionics Systems Conference (DASC). AeroMACS - an airport communications system (IEEE Seattle, USA, 2011), pp. 4-114116. doi:10.1109/DASC.2011.6095903

3. I Barhumi, G Leus, M Moonen, Optimal training design for MIMO OFDM systems in mobile wireless channels. IEEE Trans. Signal Process. 51(6), 1615-1624 (2003). doi:10.1109/TSP.2003.811243

4. S Das, P Schniter, Max-SINR ISI/ICl-shaping multicarrier communication over the doubly dispersive channel. IEEE Trans. Signal Process. 55(12), 5782-5795 (2007). doi:10.1109/TSP.2007.901660

5. Z Tang, RC Cannizzaro, G Leus, P Banelli, Pilot-assisted time-varying channel estimation for OFDM systems. IEEE Trans. Signal Process. 55(5), 2226-2238 (2007). doi:10.1109/TSP.2007.893198

6. GB Giannakis, C Tepedelenlioglu, Basis expansion models and diversity techniques for blind identification and equalization of time-varying channels. Proc. IEEE. 86(10), 1969-1986 (1998). doi:10.1109/5.720248

7. G Leus, in Proc. of EUSIPCO. On the estimation of rapidly time-varying channels (EURASIP Vienna, Austria, 2004), pp. 2227-2230

8. DK Borah, BT Hart, Frequency-selective fading channel estimation with a polynomial time-varying channel model. IEEE Trans. Commun. 47(6), 862-873 (1999). doi:10.1109/26.771343

9. H Hijazi, L Ros, Polynomial estimation of time-varying multipath gains with intercarrier interference mitigation in OFDM systems. IEEE Trans. Vehicular Technol. 58(1), 140-151 (2009). doi:10.1109/TVT.2008.923653

10. T Zemen, CF Mecklenbräuker, Time-variant channel estimation using discrete prolate spheroidal sequences. IEEE Trans. Signal Process. 53(9), 3597-3607 (2005). doi:10.1109/TSP.2005.853104

11. KAD Teo, S Ohno, in IEEE Global Telecommunications Conference, 2005. GLOBECOM '05, vol. 6. Optimal MMSE finite parameter model for doubly-selective channels (IEEE St. Louis, USA, 2005), pp. 3503-3507. doi:10.1109/GLOCOM.2005.1578424

12. P Suárez-Casal, JA García-Naya, L Castedo, M Rupp, in IEEE 14th Workshop on Signal Processing Advances in Wireless Communications. SPAWC'13. KLT-based estimation of rapidly time-varying channels in MIMO-OFDM systems (IEEE Darmstadt, Germany, 2013)

13. A Stamoulis, SN Diggavi, N Al-Dhahir, Intercarrier interference in MIMO OFDM. IEEE Trans. Signal Process. 50(10), 2451-2464 (2002). doi:10.1109/TSP.2002.803347

14. X Ma, GB Giannakis, S Ohno, Optimal training for block transmissions over doubly selective wireless fading channels. IEEE Trans. Signal Process. 51(5), 1351-1366 (2003). doi:10.1109/TSP.2003.810304

15. F Hlawatsch, G Matz, Wireless Communications Over Rapidly Time-Varying Channels. (Academic Press, USA, 2011)

16. H Hijazi, L Ros, G Jourdain, in Proceedings European Wireless Conference. OFDM channel parameters estimation used for $\mathrm{ICI}$ reduction in time-varying multipath channels (IEEE Paris, France, 2007)

17. M Meidlinger, M Simko, Q Wang, M Rupp, in IEEE International Conference on Communications 2013. ICC 2013. Channel estimators for LTE-A downlink fast fading channels (IEEE Stuttgart, Germany, 2013)

18. J Rodríguez-Piñeiro, P Suárez-Casal, JA García-Naya, L Castedo, C Briso-Rodríguez, JI Alonso-Montes, in IEEE 8th Sensor Array and Multichannel Signal Processing Workshop (SAM 2014). Experimental validation of ICl-aware OFDM receivers under time-varying conditions (IEEE A Coruña, Spain, 2014)

19. PS Casal, J Rodríguez-Piñeiro, JA García-Naya, L Castedo, in The Eleventh International Symposium on Wireless Communication Systems (ISWCS) Experimental assessment of WiMAX transmissions under highly time-varying channels (IEEE Barcelona, Spain, 2014)

20. W Nam, YH Lee, Preamble-based cell identification for cellular OFDM systems. IEEE Trans. Wireless Commun. 7(12), 5263-5267 (2008). doi:10.1109/T-WC.2008.071426

21. A Carro-Lagoa, P Suarez-Casal, J Garcia-Naya, P Fraga-Lamas, L Castedo, A Morales-Mendez, Design and implementation of an OFDMA-TDD physical layer for WiMAX applications. EURASIP J. Wireless Commun. Netw. 2013(1), 243 (2013). doi:10.1186/1687-1499-2013-243

22. $Y \mathrm{Li}, \mathrm{L} \mathrm{J}$ Cimini, Bounds on the interchannel interference of OFDM in time-varying impairments. IEEE Trans. Commun. 49(3), 401-404 (2001). doi:10.1109/26.911445

23. X Cai, GB Giannakis, Bounding performance and suppressing intercarrier interference in wireless mobile OFDM. IEEE Trans. Commun. 51(12), 2047-2056 (2003). doi:10.1109/TCOMM.2003.820752 
24. J Rodríguez-Piñeiro, JA Garcia-Naya, A Carro-Lagoa, L Castedo, in Proc. of 16th Euromicro Conference on Digital System Design. A testbed for evaluating LTE in high-speed trains (IEEE Santander, Spain, 2013)

25. Ettus USRP B210 (2013). https://www.ettus.com/product/details/UB210KIT

26. Analog Devices AD9361 RFIC (2013). http://www.analog.com/en/rfifcomponents/rfif-transceivers/ad9361/products/product.html

27. Mini-Circuits high-power amplifier TVA-11-422 (2013). http://www. minicircuits.com/pdfs/TVA-11-422.pdf

28. Interline SECTOR IS-G14-F2425-A120-V vertically polarized antenna (2014). http://interline.pl/antennas/SECTOR-V120-14dBi-120deg-2.4-2.5G Gz

29. Ubiquity AM-2G15-120 cross polarized antenna (2013). http://dl.ubnt. com/datasheets/airmaxsector/airMAX_Sector_Antennas_DS.pdf

30. M Matthaiou, DI Laurenson, C-X Wang, in IEEE Wireless Communications and Networking Conference, 2008. WCNC 2008. Capacity study of vehicle-to-roadside MIMO channels with a line-of-sight component (IEEE Las Vegas, USA, 2008), pp. 775-779. doi:10.1109/WCNC.2008.142

Submit your manuscript to a SpringerOpen ${ }^{\circ}$ journal and benefit from:

- Convenient online submission

- Rigorous peer review

- Immediate publication on acceptance

- Open access: articles freely available online

- High visibility within the field

- Retaining the copyright to your article

Submit your next manuscript at $>$ springeropen.com 